\title{
1 Intact reading ability in spite of a spatially distributed visual 2 word form 'area' in an individual born without the left 3 superior temporal lobe
}

4

5

\section{Authors}

${\mathrm{Jin} \mathrm{Li}^{1} \text {, Evelina Fedorenko }}^{2}$, Zeynep Saygin ${ }^{1 *}$

Affiliations

${ }^{1}$ Department of Psychology, The Ohio State University, Columbus, OH, USA.

${ }^{2}$ Department of Brain and Cognitive Sciences / McGovern Institute for Brain Research, MIT, Cambridge, MA, USA

*Correspondence to: 1i.9361@ osu.edu and saygin.3@osu.edu

\section{Acknowledgements}

We would like to thank our participant - EG - for her time and willingness to participate in brain research. We thank Greta Tuckute, Alexander Paunov, and Benjamin Lipkin for their help in coordinating the collaboration as well as their suggestions for data analysis. We also thank Idan Blank and Zachary Mineroff for help with EG's fMRI and behavioral data collection and members of Saygin Developmental Cognitive Neuroscience Lab for helping with control data collection. We would like to acknowledge the Athinoula A. Martinos Imaging Center at the McGovern Institute for Brain Research, MIT and the support from Center for Cognitive and Behavioral Brain Imaging (CCBBI) and Ohio Supercomputer Center (OSC). E.F. was supported by NIH awards R01 DC016607, R01 DC016950, and research funds from the McGovern Institute for Brain Research and the Department of Brain and Cognitive Sciences at MIT. Z.M.S. was supported by the Alfred P. Sloan Foundation and OSU's Arts \& Sciences College and Chronic Brain Injury initiative.

\section{Conflict of interest}

The authors declare no competing financial interests. 


\begin{abstract}
The visual word form area (VWFA) is an experience-dependent brain region in the left ventral

36 temporal cortex of literate adults that responds selectively to visual words. Why does it emerge

37 in this stereotyped location? Past research has shown that the VWFA is preferentially connected

38 to the left-lateralized frontotemporal language network. However, it remains unclear whether the

39 presence of a typical language network and its connections with ventral temporal cortex (VTC)

40 are critical for the VWFA's emergence, and whether alternative functional architectures may

41 support reading ability. We explored these questions in an individual (EG) born without the left

42 superior temporal lobe but exhibiting normal reading ability. Using fMRI, we recorded brain

43 activation to visual words, objects, faces, and scrambled words in EG and neurotypical controls.

44 We did not observe word selectivity either in EG's right homotope of the VWFA (rVWFA) - the

45 most expected location given that EG's language network is right-lateralized — or in her spared

46 left VWFA (IVWFA), in the presence of typical face selectivity in both the right and left

47 fusiform face area (rFFA, lFFA). Interestingly, multivariate pattern analyses revealed voxels in

48 EG's rVWFA and IVWFA that showed 1) higher within- than between-category correlations for

49 words (e.g., Words-Words>Words-Faces), and 2) higher within-category correlations for words

50 than other categories (e.g., Words-Words>Faces-Faces). These results suggest that a typical left-

51 hemisphere language network may be necessary for the emergence of focal word selectivity

52 within ventral temporal cortex, and that orthographic processing may depend on a distributed

53 neural code, which appears capable of supporting reading ability.
\end{abstract}

55 Keywords: lesion study; Visual Word Form Area (VWFA); brain plasticity; word selectivity;

56 multivariate pattern analysis (MVPA); category selectivity 


\section{Introduction}

58 In the past two decades, numerous regions in the ventral temporal cortex (VTC) have been

59 identified and characterized that respond selectively to different high-level visual categories (e.g.,

60 faces: Kanwisher et al., 1997; places: Epstein \& Kanwisher, 1998; bodies: Downing et al., 2001;

61 for review, see Kanwisher, 2010). What are the origins of these specialized regions? How do

62 human brains develop this functional topography? Some have hypothesized that the functional

63 organization of the VTC may be innate and related to the evolutionary importance of certain

64 high-level visual categories. Indeed, face perception and recognition abilities appear to be

65 heritable (Wilmer et al., 2010; Zhu et al., 2010), and 4-6 month-old infants already show face-

66 and place-responsive areas within expected locations in the VTC (Deen et al., 2017). A related

67 hypothesis is that pre-existing biases for certain visual attributes (e.g., retinotopy, Hasson et al.,

68 2002; Malach et al., 2002; rectilinearity, Nasr et al., 2014) or perceptual dimensions (e.g., real-

69 world size and animacy, Konkle \& Caramazza, 2013) may predispose a brain region to become

70 selective for particular visual categories. However, evolutionary pressures cannot explain the

71 existence of a brain region that specializes for orthography (Hannagan et al., 2015) — the visual

72 word form area (VWFA). The VWFA is a small region in the left lateral VTC that shows strong

73 selectivity for visual words and letter strings in literate individuals (e.g., L. Cohen et al., 2003;

74 Dehaene \& Cohen, 2011; Hamamé et al., 2013). Perhaps surprisingly_given its late

75 emergence-the VWFA is located in approximately the same location across individuals and

76 languages (Baker et al., 2007). What sets word forms apart from other visual categories and why

77 does the VWFA land in this stereotyped location? 
79 One compelling possibility is that the specialization of category-selective regions in the VTC is

80 constrained by their differential connectivity to the rest of the brain (the Connectivity Hypothesis;

81 Mahon \& Caramazza, 2011; Martin, 2006). Indeed, previous work showed that category-

82 selective responses can be predicted from both structural and functional connectivity (Osher et

83 al., 2015; Saygin et al., 2012); further, these distinct connectivity patterns may already exist at

84 birth and may drive future functional specialization (e.g., newborns show functional connectivity

85 differences between lateral VTC which houses the VWFA vs. medial VTC: Barttfeld et al.,

86 2018). Thus, written words may be processed in a stereotyped region within the left VTC due to

87 this region's pre-existing connectivity with the left-lateralized language network (e.g., Behrmann

88 \& Plaut, 2013; Dehaene et al., 2015; Martin, 2006). This network consists of left lateral frontal

89 and lateral temporal areas and selectively supports language comprehension and production (e.g.,

90 Fedorenko et al., 2011).

91

92 Consistent with this idea, a number of studies have reported both anatomical and functional

93 connections between the VWFA and the language network in neurotypical adults. For example,

94 compared to the adjacent FFA, the VWFA shows stronger anatomical connectivity to the left

95 superior temporal, anterior temporal, and inferior frontal areas (perisylvian putative language

96 regions) (Bouhali et al., 2014). Several candidate white matter fascicles may serve to connect the

97 VWFA with frontal and superior temporal language cortex (Wandell et al., 2012; Yeatman \&

98 Feldman, 2013). Similarly, using resting-state functional connectivity, Stevens et al. (2017)

99 found that the individually defined VWFA connects to the posterior left inferior frontal gyrus as

100 well as the left planum temporale, both part of the distributed left language network. 
103 Moreover, a longitudinal study in children showed that the location of the VWFA could be

104 successfully predicted by its connectivity patterns at the pre-reading stage (before any word

105 selectivity within the VTC is observed), and that the most predictive connections of the relevant

106 area of the VTC were with the frontal and temporal language areas (Saygin et al., 2016). Even

107 more strikingly, this pattern of preferential connectivity appears to be already present in neonates

108 (Li et al., 2020). These connections between the VWFA and the frontotemporal language

109 network appear to be functionally important such that their damage or stimulation leads to

110 reading difficulties. For example, a case study of a child with a missing arcuate fasciculus (AF,

111 presumably connecting the VTC and other parts of the temporal cortex to parietal and frontal

112 areas; Wandell et al., 2012) found impaired reading ability (Rauschecker et al., 2009). Similarly,

113 a lesion in the white matter ventral to the angular gyrus resulted in alexia without agraphia,

114 presumably disrupting the VWFA's connections with the lateral temporal language areas

115 (Greenblatt, 1976).

117 A different way to assess the importance of a language network in developing visual word

118 selectivity is to ask whether language regions and the VWFA occupy the same hemisphere. In

119 the majority of individuals, the language regions and the VWFA co-lateralize to the left

120 hemisphere (LH) (e.g., Cai et al., 2010; Gerrits et al., 2019). In rare instances where neurotypical

121 individuals show right-hemispheric $(\mathrm{RH})$ language dominance, VTC activation during reading

122 tasks also tends to be right-lateralized (e.g., Cai et al., 2008; Van der Haegen et al., 2012).

123 Another population where the language network is right-lateralized are individuals with

124 congenital or early left hemisphere (LH) damage (e.g., Asaridou et al., 2020). In the presence of 
125 early LH damage, linguistic abilities tend to develop normally (e.g., Newport et al., 2017; Staudt,

126 2010; Staudt et al., 2001; see François et al., 2021 for a review). However, little is known about

127 the effects of early LH damage on reading ability and on the neural architecture of visual word

128 processing. In particular, if the left VTC is completely deafferentiated from the downstream LH

129 language cortex at birth, does the VWFA emerge in the right VTC when the language network is

130 forcibly remapped to the right hemisphere, or does it still emerge in the left VTC, due to some

131 pre-existing bias (e.g., innate connectivity with any spared LH cortex)? Indeed, some previous

132 studies show that language processing and reading may engage opposite hemispheres (e.g., Van

133 der Haegen et al., 2012). Or_perhaps even more drastically_does the VWFA look atypical

134 (e.g., smaller or less functionally selective), whatever hemisphere it emerges in? Do these

135 potential differences affect selectivity for other high-level visual categories in the VTC? And if

136 the VWFA manifests atypically but reading ability is normal, what does this tell us about the

137 implementation-level flexibility with respect to orthographic processing?

139 Here we investigate possible functional reorganization of the visual word selectivity in the

140 absence of a typical left-lateralized language network. We have a unique opportunity to examine

141 fMRI responses to stimuli from different visual categories in an individual (EG) born without the

142 left superior temporal lobe (likely due to pre/perinatal stroke) but with IVTC largely intact. EG's

143 frontotemporal language network is completely remapped to the right hemisphere; no language-

144 related responses, as assessed with fMRI, were observed in the remaining parts of EG's left

145 hemisphere (Tuckute et al., 2021). EG's reading abilities (as well as other linguistic abilities) are

146 intact. We here investigated a) whether, in the presence of a right-lateralized language network, a

147 typical VWFA would emerge in the right VTC (in a typical location, or perhaps in other parts of 
148 the right VTC), and b) whether any word-selectivity is observed in the (spared) left VTC. To

149 foreshadow the results, no word selectivity was observed in EG's right or left VTC, in the

150 presence of typical selectivity for other visual categories. We then explored the possibility that

151 orthographic processing is supported by still selective but more distributed neural populations

152 using multivariate analyses, and indeed observed such multivariate selectivity bilaterally, but

153 manifesting more strongly in the right VTC.

\section{Methods}

\section{Participants}

157 Critical participant. The participant EG (fake initials; right-handed female with an advanced

158 professional degree, 54 year-old at the time of testing) contacted Dr. Fedorenko's lab to

159 participate in brain research studies. Based on her own report, the lack of the left superior

160 temporal lobe (Figure 1) was discovered when she was 25 years old (in her first MRI scan in

161 1987) and being treated for depression. No known head traumas or injuries were reported as a

162 child or adult. Several medical MRI scans were performed in subsequent years (1988, 1998, and

163 2013) and no change was observed compared to the initial scans. Importantly, EG did not report

164 any difficulties in reading or general language abilities (see details below). She had also acquired

165 fluency in a second language (Russian). EG was invited to participate in a series of behavioral

166 and fMRI assessments at MIT in October 2016. Written informed consent was obtained from EG,

167 and the study was approved by MIT's Committee on the Use of Humans as Experimental

168 Subjects (COUHES). 
170 To formally evaluate EG's linguistic abilities, five standardized language assessment tasks were

171 administered: i) an electronic version of the Peabody Picture Vocabulary Test (PPVT-IV) (Dunn

172 \& Dunn, 2007); ii) an electronic version of the Test for Reception of Grammar (TROG-2)

173 (Bishop, 2003); iii) the Western Aphasia Battery-Revised (WAB-R) (Kertesz, 2006); iv) the

174 reading and spelling components of PALPA (Kay et al., 1992); and v) an electronic version of

175 the verbal components of the Kaufman Brief Intelligence Test (KBIT-2) (Kaufman \& Kaufman,

176 2004). PPVT- IV and TROG-2 target receptive vocabulary and grammar, respectively. In these

177 tasks, the participant is shown sets of four pictures accompanied by a word (PPVT-IV, 72 trials)

178 or sentence (TROG-2, 80 trials) and has to choose the picture that corresponds to the

179 word/sentence by clicking on it. WAB-R (Kertesz, 2006) is a more general language assessment

180 developed for persons with aphasia. It consists of 9 subscales, assessing 1) spontaneous speech, 2)

181 auditory verbal comprehension, 3) repetition, 4) naming and word finding, 5) reading, 6) writing,

182 7) apraxia, 8) construction, visuospatial, and calculation tasks, and 9) supplementary writing and

183 reading tasks. The verbal components of KBIT-2 include 1) the Verbal Knowledge subtest,

184 which consists of 60 items measuring receptive vocabulary and general information about the

185 world, and 2) the Riddles subtest consists of 48 items measuring verbal comprehension,

186 reasoning, and vocabulary knowledge. Most relevant to the current investigation, the reading

187 component of WAB-R includes comprehension of written sentences and reading commands; the

188 supplementary reading tasks include reading of irregular words and nonwords. The reading

189 component of PALPA (Kay et al., 1992) consists of 21 tasks (tasks \#18 through 38), which focus

190 on single letter recognition, visual lexical decision, out-loud reading (of words with diverse

191 lexical properties and sentences), and homophone definition (see Supplementary Table 2 for

192 details). 
194 In line with her self-report, EG performed within normal range on all language assessment tasks.

195 She got $90 \%$ correct on PPVT, 99\% correct on TROG-2, 97.6, 98.6, and 98.4 on the aphasia,

196 language, and cortical quotients of the WAR-B (the criterion cut-off score for diagnosis of

197 aphasia is an aphasia quotient of 93.8), and a score of 130 (98th percentile) on the KBIT-verbal.

198 EG's performance was therefore not distinguishable from the performance of neurotypical

199 controls. With respect to the reading assessments, EG made no errors on the main reading

200 component of WAB-R, no errors in the reading of irregular words, and one error in the reading

201 of nonwords (reading 'gobter' instead of 'globter'). For the PALPA tasks, she made no errors on

202 tasks that focus on single letters (tasks \#18-23), no errors on the visual decision tasks (tasks \#24-

203 27), no errors on the out-loud reading tasks (tasks \#29-37), and no errors on the homophone

204 definition task (task \#38). For the homophone decision task (task \#28), EG made three errors

205 (out of the 60 trials; all were made on nonword pairs: she did not judge the following pairs as

206 sounding the same: heem-heam, byme-bime, and phex-feks). Based on this comprehensive

207 assessment, EG appears to have intact visual letter and word recognition ability.

209 With respect to fMRI tasks that relevant to the current study, EG completed five runs of the

210 VWFA localizer; see The VWFA localizer task section below).

212 Neurotypical controls. Twenty-five adults (11 female, mean age $=23.6$ years old; age range 18-

21338 years; standard deviation 5.21 years) from The Ohio State University (OSU) and the

214 surrounding community were included in the present study. As part of ongoing projects

215 exploring the relationship between brain function and connectivity, all participants completed a 
216 battery of fMRI tasks, including, critically, the same VWFA localizer task that EG completed

217 (see The VWFA localizer task section below). All participants had normal or corrected-to-normal

218 vision, and reported no neurological, neuropsychological, or developmental diagnoses. Written

219 informed consent was obtained from all participants and the study was approved by Institutional

220 Review Board at OSU. (It is worth noting that although the control group participants were

221 younger than EG, further examination revealed no significant correlation between age and word-

222 selectivity (responses to Words minus the average of the other conditions) in our control group

$223(\mathrm{r}=-0.259, \mathrm{p}=0.094 ; \mathrm{p}$-value was obtained by a permutation test $)$.

\section{Data acquisition}

226 EG's data were collected on a 3 Tesla Siemens Trio scanner with a 32-channel head coil at the

227 Athinoula A. Martinos Imaging Center at the McGovern Institute for Brain Research at MIT.

228 Data of the control group participants were acquired on a 3 Tesla Siemens Prisma scanner with a

229 32-channel head coil at the Center for Cognitive and Behavioral Brain Imaging at OSU. To

230 ensure that any differences between EG and our control group were not due to scanner

231 differences, we compared word-selectivity in the current control group to a smaller group of

232 adults recruited at MIT and scanned with the same scanner and protocols as EG; no differences

233 were observed (Supplementary Table 1). For both EG and controls, a whole-head, high

234 resolution T1-weighted magnetization-prepared rapid acquisition with gradient echo (MPRAGE)

235 scan was acquired (EG: repetition time $(\mathrm{TR})=2530 \mathrm{~ms}$, echo time $(\mathrm{TE})=3.48 \mathrm{~ms}$; voxel

236 resolution $=1.0 \mathrm{~mm}^{3}$; the control group: $\mathrm{TR}=1390 \mathrm{~ms}$, $\mathrm{TE}=4.62 \mathrm{~ms}$, voxel resolution $=1.0$

$237 \mathrm{~mm}^{3}$ ). Functional images were acquired with the same echo-planar imaging (EPI) sequence for

238 both EG and controls: TR=2000ms, TE=30ms, $172 \mathrm{TRs}, 100 \times 100$ base resolution, voxel 
239 resolution $=2.0 \mathrm{~mm}^{3}, 25$ slices approximately parallel to the base of the temporal lobe to cover

240 the entire ventral temporal cortex.

\section{The VWFA localizer task}

243 A VWFA localizer was used to define high-level category-selective regions and to measure

244 category-selective responses (see Saygin et al., 2016, for details). Briefly, black and white line

245 drawings of words, scrambled words, objects, and faces, along with the fixation condition were

246 shown in a blocked design. A grid was overlaid on top of the stimuli so that all stimulus types

247 (not just scrambled words) had edges. Each stimulus was presented for 500ms (ISI=0.193s) and

248 overlaid on a different single-color background, and 26 stimuli (including 2 repetitions) were

249 presented in each block. Each run consisted of 19 blocks ( 4 blocks per stimulus type and 3

250 fixation blocks), and participants performed a one-back task. The stimuli are available for

251 download at http://www.zeynepsaygin.com/ZlabResources.html. EG completed 5 runs, and

252 participants in the control group completed 2 runs. Note that previous studies using the same task

253 indicated that 2 runs of data are sufficient to successfully identify the VWFA in a neurotypical

254 population (Saygin et al., 2016); here, we acquired more runs for EG to ensure that we had

255 sufficient power and that the results obtained for EG were stable across runs (see Definition of

256 functional regions of interest and univariate analyses section for details).

258 Preprocessing and fMRI analysis

259 Data were analyzed with Freesurfer v.6.0.0 (http://surfer.nmr.mgh. harvard.edu/), FsFast

260 (https://surfer.nmr.mgh.harvard.edu/fswiki/FsFast), FSL

261 (https://fsl.fmrib.ox.ac.uk/fsl/fslwiki/FLIRT) and custom MatLab code. All structural MRI data 
262 were processed using a semiautomated processing stream with default parameters (recon-all

263 function in Freesurfer: https://surfer.nmr.mgh.harvard.edu/fswiki/recon-all/), which includes the

264 following major steps: intensity correction, skull strip, surface co-registration, spatial smoothing,

265 white matter and subcortical segmentation, and cortical parcellation. Cortical gray matter and

266 ventral temporal cortex masks were created based on the Desikan-Killiany (Desikan et al., 2006)

267 parcellation in native anatomy for each subject. The success of cortical reconstruction and

268 segmentation was visually inspected for EG.

270 Functional images were motion-corrected (time points where the difference in total vector

271 motion from the previous time point exceeded $1 \mathrm{~mm}$ were excluded), data from each run were

272 registered to each individual's anatomical brain image using bbregister, and resampled to

$2731.0 \times 1.0 \times 1.0 \mathrm{~mm}^{3}$. For EG, instead of registering the functional image of each run to the

274 anatomical brain separately, we aligned the functional images of the first four runs to the last run

275 (which had successful functional-anatomical cross-modal registration with bbregister) with linear

276 affine transformation (FLIRT); then the functional-anatomical transformation for the last run was

277 applied to all functional runs and was visually inspected (tkregisterfv) for functional to

278 anatomical alignment.

280 The preprocessed functional data were then entered into a first-level analysis. Specifically, data

281 were detrended, smoothed (3mm FWHM kernel), and the regressor for each experimental

282 condition (Words, Scrambled Words, Objects, and Faces) was defined as a boxcar function (i.e.,

283 events on/off) convolved with a canonical hemodynamic response function (a standard gamma

284 function $(d=2.25$ and $t=1.25))$. Orthogonalized motion measures from the preprocessing stage 
were used as nuisance regressors for the GLM. Resulting beta estimates for each condition and contrasts of interest between categories (i.e., Words > Others other categories and Faces > Others

287 other categories) were used in further analyses. For multivariate analyses, no spatial smoothing 288 was applied.

\section{Definition of functional regions of interest and univariate analyses}

291 The subject-specific functional regions of interests (fROIs) were defined with a group-

292 constrained subject-specific (GcSS) approach (Fedorenko et al., 2010). In this approach,

293 individual fROIs are defined by intersecting participant-specific fMRI contrast maps for the

294 contrast of interest (e.g., Words > Other conditions) with some spatial constraint(s) or 'parcel(s)',

295 which denote the area(s) in the brain within which most individuals show responses to the

296 relevant contrast (based on past studies with large numbers of participants). In the present study,

297 the VWFA and the FFA parcels were derived from previous studies (FFA, Julian et al., 2012;

298 VWFA, Saygin et al., 2016) (Figure 1) and were generated based on probabilistic maps of

299 functional activation for the relevant contrasts in independent groups of participants. We

300 registered these parcels to our participants' own anatomy using the combined volume and

301 surface-based (CVS) non-linear registration method (mri_cvs_register; Postelnicu et al., 2009).

302 After mapping the functional parcels to each participant's brain, we defined the VWFA fROI by

303 selecting within the VWFA parcel the top $10 \%$ of most active voxels for the contrast Words >

304 Other conditions (i.e., scrambled words, objects, and faces). Similarly, we defined the FFA by

305 selecting within the FFA parcel the top $10 \%$ of most active voxels for the contrast Faces $>$ Other

306 conditions. For the control participants, we used run 1 to define the fROIs, and the run 2 to

307 extract percent signal changes (PSCs; beta estimates divided by baseline activation) for each of 
308 four experimental conditions. For EG, who had 5 runs of data, this procedure was performed

309 iteratively for every 2-run/3-run split (e.g., defining fROIs with runs $1 \& 2$ and extracting PSCs

310 from runs 3-5, defining with runs $1 \& 3$ and extracting PSCs from runs 2, 4, and 5, etc.; 10

311 combinations in total), and the results were averaged to derive a single estimate per condition per

312 fROI. Note that although the parcels are relatively large, by design (in order to accommodate

313 inter-individual variation in the precise location of the functional region), and can overlap, the

314 resulting fROIs within an individual are small and do not overlap (Saygin et al., 2016).

\section{Multivariate analyses: Split-half correlations}

317 To further examine whether visual words may be represented and processed in a spatially

318 distributed manner, we performed a multivariate pattern analysis (MVPA) to measure distinctive

319 activation patterns for different conditions. The analyses were performed with CoSMoMVPA

320 toolbox (Oosterhof et al., 2016) (https://www.cosmomvpa.org/). In line with the approach

321 introduced in Haxby et al. (2001), we examined split-half within-category and between-category

322 correlations. In particular, a searchlight (radius = 3 voxels) was created for each voxel within the

323 VTC, and response patterns (i.e., beta estimates for each of the four conditions, normalized by

324 subtracting mean responses across all conditions) were extracted from each searchlight.

326 Before performing the critical analysis, we asked whether the overall multivariate representation

327 structure of the VTC is typical in EG. To do so, we constructed a representational similarity

328 matrix (RSM) from pairwise similarities (i.e., correlations) based on the voxel-wise response

329 patterns in a given searchlight area (defined above) to different conditions (e.g., the correlation

330 between activation patterns across voxels to Words and Faces in a given searchlight area). 
331 Correlations of all searchlights within the VTC were then Fisher z-transformed and averaged.

332 This resulted in one 4x4 RSM from two runs of data for each participant in the control group; for

333 EG, RSMs from ten run combinations were averaged to get a single RSM. We then calculated

334 RSM similarity (correlation) between EG and controls, and tested whether this correlation was

335 different from the correlations between any given control individual and the rest of the control

336 group (within-controls correlations; similarity of RSM of each subject to the average RSM of the

337 remaining control subjects).

339 Then, similarity of response patterns within a category (e.g., Words-Words or Faces-Faces) vs.

340 between categories (e.g., Words-Faces) was calculated within the VWFA and FFA parcel

341 boundaries by Pearson's correlation in a pair-wise manner, and then Fisher z-transformed. We

342 identified voxels of interest that satisfied the following two criteria: 1) voxels whose local

343 neighborhoods showed higher within- than between-category correlations (and which therefore

344 represent categories distinctively); and 2) voxels whose local neighborhoods showed higher

345 within-condition correlations for a particular category (e.g., Words-Words) than within-condition

346 correlations for other categories (e.g., Faces-Faces). The second criterion identified voxels which

347 represent a particular category (e.g., visual words) in a more selective fashion. We refer to such

348 voxels as multivariate-selective voxels.

350 To examine hemispheric differences, we computed the number of voxels that exhibited

351 multivariate selectivity for words or faces in the two hemispheres (within the relevant parcels).

352 To control for the difference in the size of the search spaces (i.e., the parcels), we divided the

353 number of the multivariate-selective voxels by the total size of the relevant parcel. 


\section{Statistical analyses}

356 Paired t-tests were used for comparisons between conditions for EG (across ten run split

357 combinations) and within the control group. For all analyses where we compared EG's response

358 to the control group, we used a Crawford-Howell modified t-test (Crawford \& Howell, 1998),

359 which is widely used in single-case studies because it accounts for the variance of the control

360 group, and the percentage of false positives remains the same for various sizes of the control

361 sample (N=5-50) (Crawford et al., 2009). This frequentist approach provided the point estimate

362 (p-value) for the proportion of the control population that will obtain a score lower than the

363 patient's score. One-tailed tests were reported for the critical (directional) hypothesis tested in

364 the current study (unless noted otherwise). In addition, we computed the Bayesian 95\% credible

365 interval (a Bayesian alternative to the modified t-test; Crawford \& Garthwaite, 2007) to

366 demonstrate the range of p-values based on 10,000 iterations. To assess the significance of RSM

367 correlations between EG and controls, we generated a null distribution of correlation values by

368 shuffling the matrix of EG and controls (i.e., randomizing the labels of values in the RSMs) and

369 then correlating the new shuffled matrices. This procedure was repeated 10,000 times to create

370 the null distribution of the correlation values. The p-value was calculated by counting the

371 number of correlations in the null-distribution that were higher than the correlation value based

372 on the correct category labels, and then divided by 10,000 .

373

374 Results

375 Is word selectivity remapped to the right hemisphere when the language network is remapped to

376 the right hemisphere from birth? 
378 We first examined univariate word selectivity in EG's rVWFA. With the VWFA localizer, we

379 defined the individual-specific rVWFA fROIs, in EG and controls, within a spatial constraint

380 (rVWFA parcel; see Figure 1 and Methods) by contrasting Words versus all other categories

381 (Words > Others). We then extracted the activation to all four conditions from independent data

382 (see Methods for details). In neurotypical literate individuals, word selectivity is strongly left-

383 lateralized (McCandliss et al., 2003); selective responses to visual words in the right homotope

384 of IVWFA are less frequently observed and are less spatially consistent across individuals.

385 Results from our control group are in line with this picture: we found no selective activation to

386 Words as compared to other visual categories in the rVWFA (Words vs. Scrambled Words:

$387 \mathrm{t}(24)=-0.964, \mathrm{p}=0.345$; Words vs. Objects: $\mathrm{t}(24)=-0.780, \mathrm{p}=0.443$; Words vs. Faces: $\mathrm{t}(24)=-$

388 0.523, $\mathrm{p}=0.605$; Figure 2A).

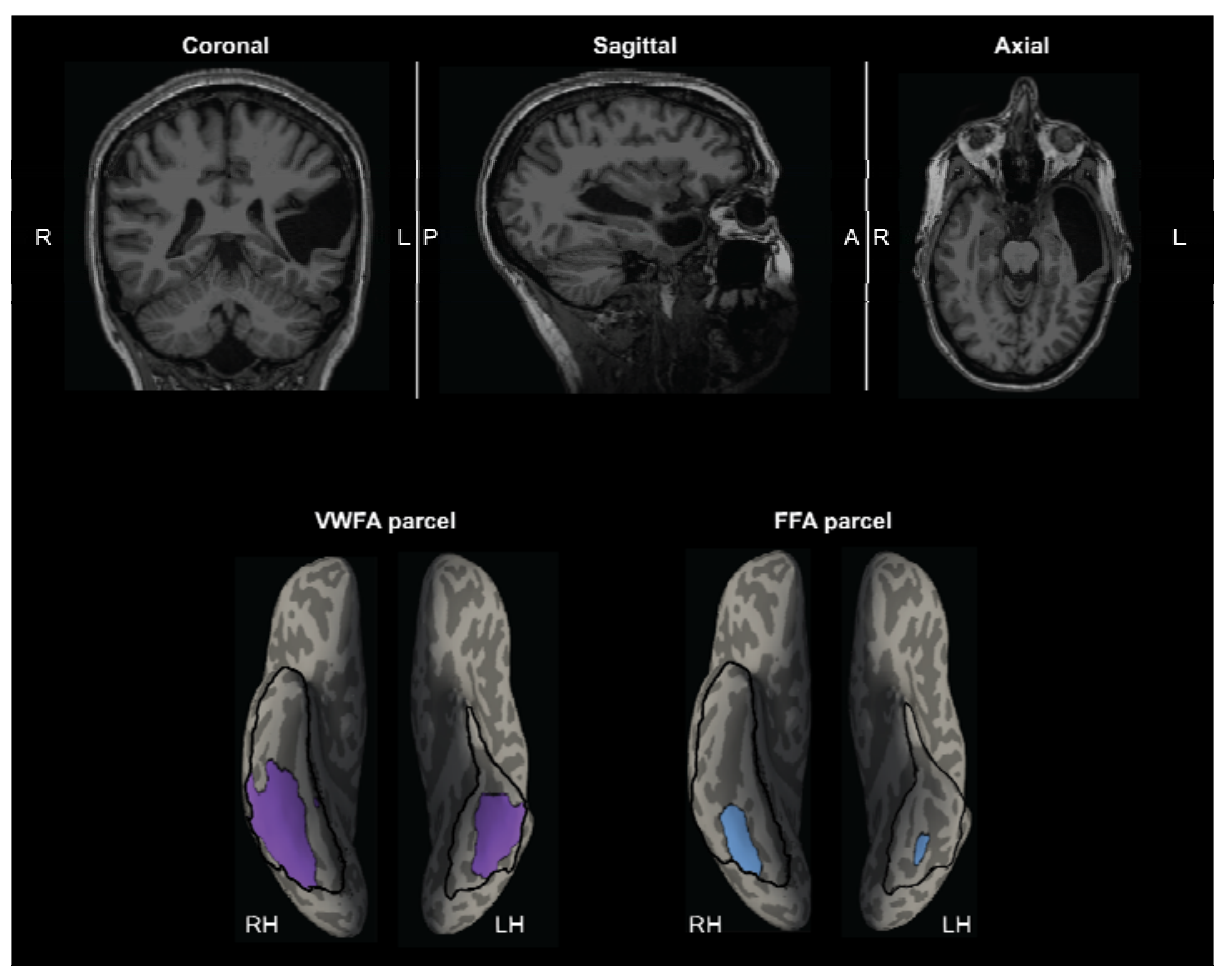


392 Figure 1. EG's MRI showing the missing cortex and the parcels for the right and left VWFA and FFA.

393 Top, T1-weighted images in coronal, sagittal, and axial views. Bottom, the VWFA (purple) and FFA

394 (blue) parcels are projected on EG's reconstructed surface. The parcels served as spatial constraints in defining the fROIs (see Methods), but we also explored the entire VTC for category selectivity (outlined with black solid lines). By design, the parcels are relatively large (to accommodate inter-individual variability in the precise locations of these areas) and therefore can overlap, but the individual fROIs are small and do not overlap. Note that even though part of the anterior IVTC is missing in EG, the

Critically, in EG, whose language network is completely reorganized to the right hemisphere

(Figure 2B). To ensure that this result is not due to the choice of a particular threshold (i.e., top $10 \%$ ) used to define the rVWFA fROI, we performed the same analysis as above at a range of

411 then compared EG to the controls, and found that there was a trend whereby responses to Words

412 (relative to baseline) were slightly higher in EG compared to the control group $(\mathrm{t}(24)=0.503, \mathrm{p}=$

4130.310 , modified t-test; 95\% Bayesian CI [0.17, 0.45]), but this difference was not significant.

416 examined face selectivity in EG's rFFA, which is spatially proximal to the rVWFA. Similar to

417 the rVWFA analysis above, we defined the rFFA fROI by contrasting Faces versus other 418 categories (Faces > Others), and extracted the activation to the four conditions from independent 
419 data (see Methods for details). EG's face selectivity remained intact (Figure 2E) and did not

420 differ from that of the control group (Figure 2D; $\mathrm{t}(24)=-0.031, \mathrm{p}=0.488$, modified $\mathrm{t}-\mathrm{test}$,

421 Bayesian 95\% CI of EG's activation [0.35 0.50]). EG's rFFA fROI showed significantly higher

422 responses to Faces than to other conditions (Faces vs. Words: $\mathrm{t}(9)=24.813, \mathrm{p}=1.346 \times 10^{-9}$; Faces

423 vs. Scrambled Words: $\mathrm{t}(9)=16.850, \mathrm{p}=4.092 \times 10^{-8}$; Faces vs. Objects: $\mathrm{t}(9)=20.312, \mathrm{p}=7.921 \times 10^{-9}$;

424 Figure 2E), just like what we observed in the control group (Faces vs. Words: $\mathrm{t}(24)=15.299$, $\mathrm{p}=$

$4257.054 \times 10^{-14}$; Faces vs. Scrambled Words: $\mathrm{t}(24)=12.915, \mathrm{p}=2.689 \times 10^{-12}$; Faces vs. Objects:

$426 \mathrm{t}(24)=10.724, \mathrm{p}=1.235 \times 10^{-10}$; Figure 2D). Moreover, the face selectivity in EG's rFFA was

427 observed across all thresholds used to define the rFFA (Figure 2F).
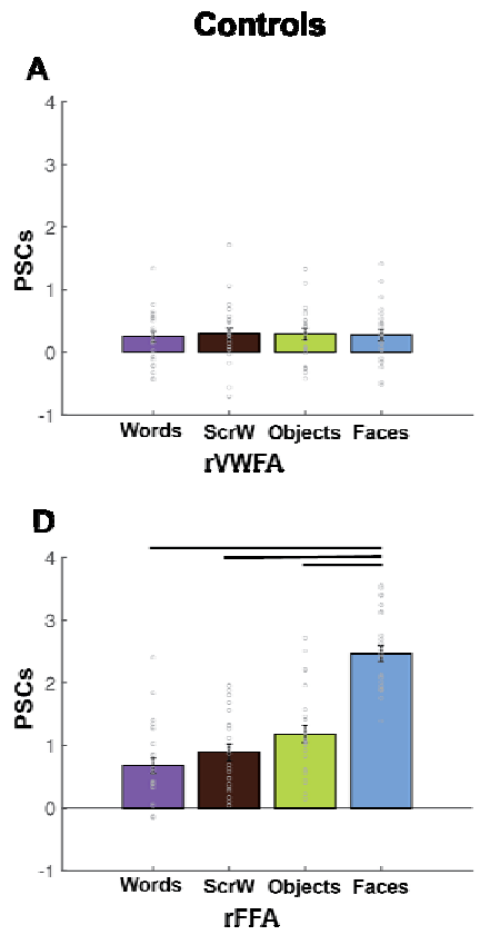

430

431

432
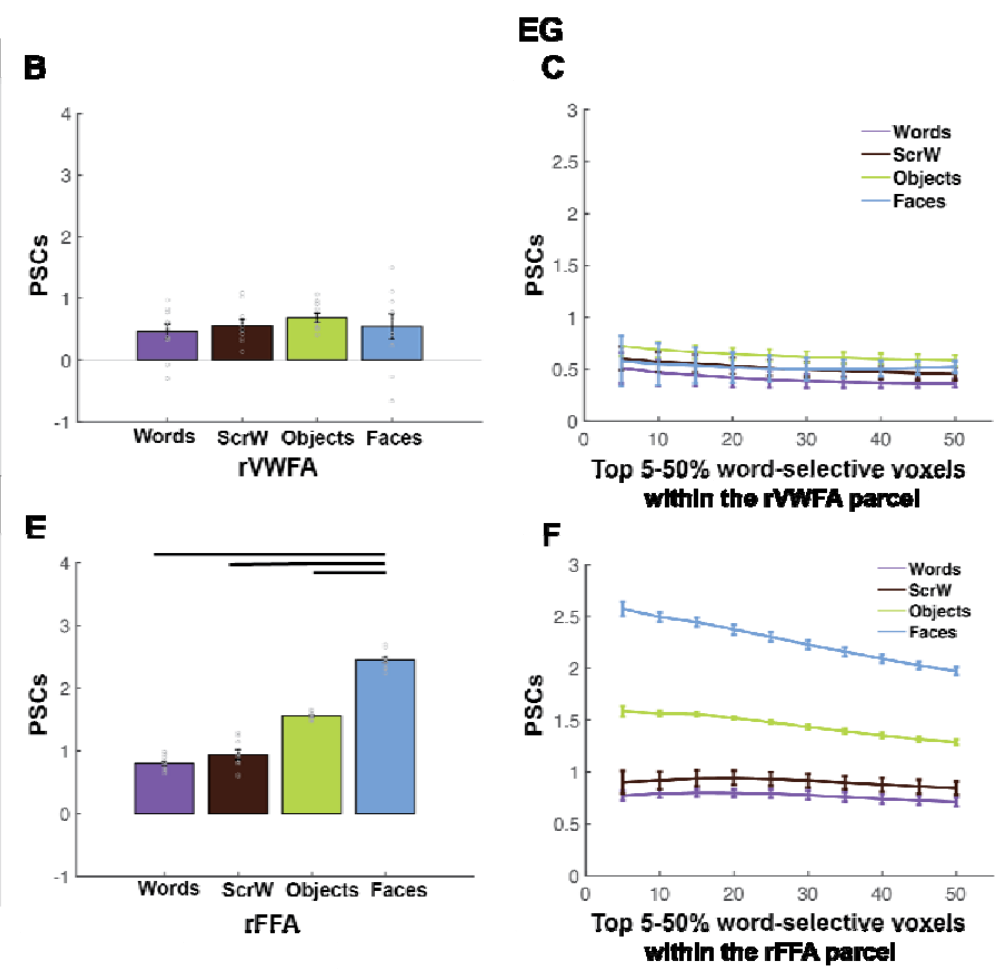
Figure 2. Responses to four conditions in the rVWFA and rFFA for EG and the control group. (A) Bar plots show mean PSCs to the four conditions estimated in independent data within individually defined rVWFA fROIs (i.e., top 10\% word-selective voxels within the rVWFA parcel) for the control group. (B) Mean PSCs to the four conditions estimated in independent data within the individually defined rVWFA fROI for EG. Here and in E, the results are averaged across run combinations. (C) Parametrically decreasing the threshold for defining the rVWFA fROI from the top 5\% to 50\% word-selective voxels within the rVWFA parcel. Number of voxels in the rVWFA fROIs: $5 \%=384$ voxels, $50 \%=3007$ voxels. Here and in F, average PSCs across run combinations are shown for each threshold. (D) Mean PSCs to the four conditions estimated in independent data within individually defined rFFA fROIs for the control group. (E) Mean PSCs to the four conditions estimated in independent data within the individually defined rFFA fROI for EG. (F) Parametrically decreasing the threshold for defining the rFFA fROI from the top 5\% to 50\% face-selective voxels within the rFFA parcel. Number of voxels in the defined rFFA fROIs: $5 \%=90$ voxels, $50 \%=699$ voxels. In the bar plots, dots correspond to individual data points for each condition (controls: $n=25$ participants; EG: $n=10$ run combinations, from ten iterations). Horizontal bars reflect significant paired t-tests $\mathrm{p}<0.05$. Error bars in both the bar and line plots denote standard errors of the mean by participants (for the control group) and by run combinations (for EG).

To ensure that we did not miss any possible word-selective voxels by applying a predefined

452 spatial constraint (i.e., VWFA parcel) and to account for the possibility that EG's VWFA is

453 located in a different part of the visual cortex, we searched for word selectivity within the entire

454 rVTC mask for EG. Specifically, different thresholds from top $1 \%$ to top $10 \%$ were used to

455 define the most word-selective voxels (Words > Others). Even within this broad mask, no word-

456 selective responses were observed in independent data across all thresholds; in fact, the

457 responses were lowest to words than the other three conditions (Figure 3A). In contrast, robust

458 face-selective responses were observed in independent data across all thresholds when searching

459 for face-selective voxels (Faces > Others) (Figure 3B).

461 Finally, we supplemented our main analyses, which rely on the Words > Others contrast to

462 define the VWFA, as is commonly done in the literature (e.g., Dehaene-Lambertz et al., 2018;

463 Rosenke et al., 2021), with an analysis, which relies on a less stringent localizer contrast (Words

464 vs. Scrambled Words; Glezer et al., 2009; Lerma-Usabiaga et al., 2018). Unlike the Words > 
Others contrast, this contrast does not control for semantics or visual stimulus complexity. Even

A

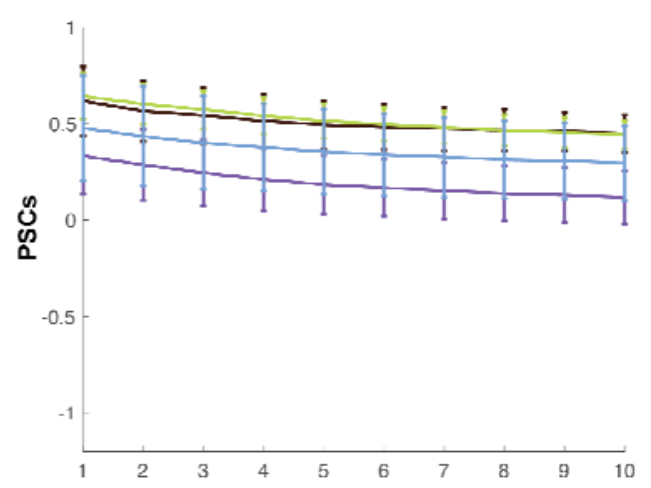

Top 1-10\% word-selective voxels within the rVTC

c

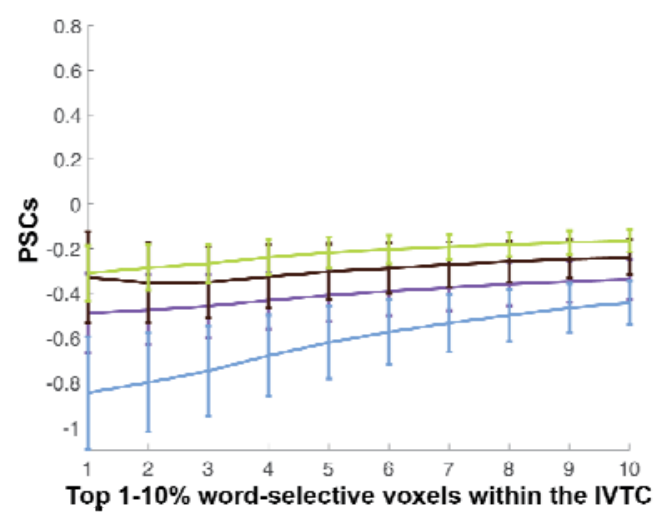

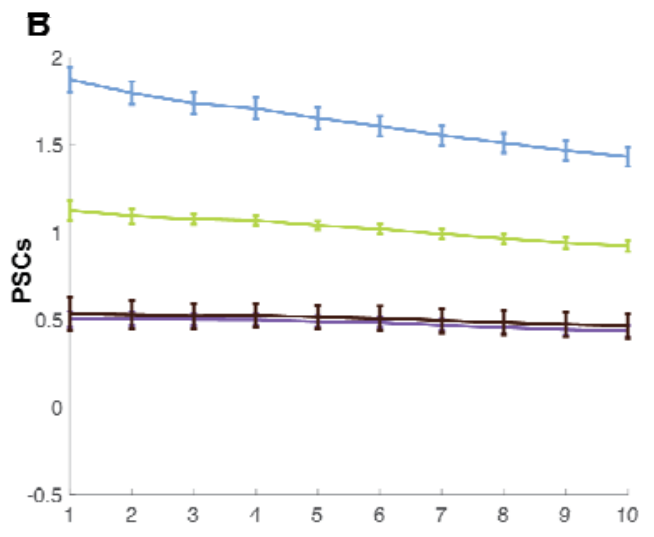

Iop $1-10 \%$ face-selective voxels within the rVTC

D

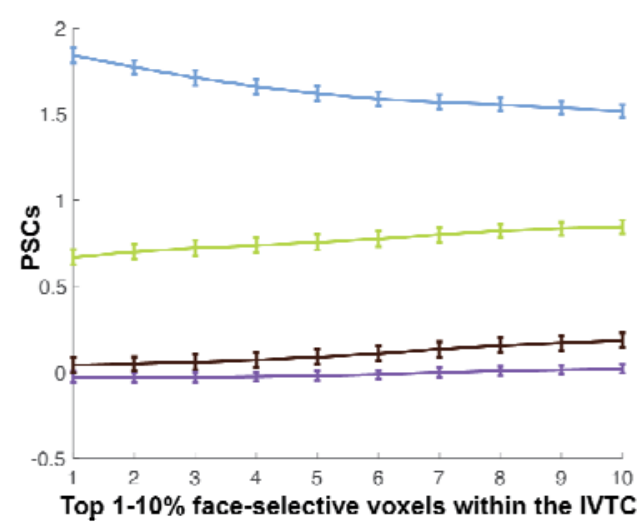

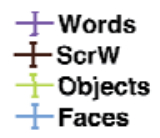

Figure 3. Mean PSCs in the rVTC and IVTC at different thresholds for EG. (A-B) Parametrically decreasing the threshold for defining word-selective (Words $>$ Others) and face-selective (Faces $>$ Others) voxels from the top $1 \%$ to $10 \%$ within the rVTC. Mean PSCs across run combinations (from 10 iterations) are shown for each threshold. (C-D) Parametrically decreasing the threshold for defining word-selective and face-selective voxels from the top $1 \%$ to $10 \%$ within the IVTC. Mean PSCs across run combinations are shown for each threshold. Number of selected voxels: rVTC: $1 \%=216$ voxels, $10 \%=2164$ voxels; IVTC: $1 \%=154$ voxels, $10 \%=1536$ voxels. 
Because we did not observe a right-lateralized VWFA and because the left VTC was largely intact, we also asked whether a canonical IVWFA may have developed in EG (perhaps due to some specific visual features of word forms that are better represented in the left

483 hemisphere/right visual field (e.g., Hsiao \& Lam, 2013; Seghier \& Price, 2011; Tadros et al., 484 2013). In the control group, we observed robust word selectivity in the IVWFA (as expected

485 from previous studies), with Words eliciting greater activation than each of the other conditions:

486 Words vs. Scrambled Words: $\mathrm{t}(24)=5.413, \mathrm{p}=1.467 \times 10^{-5}$; Words vs. Objects: $\mathrm{t}(24)=4.077, \mathrm{p}=$ $4874.333 \times 10^{-4} ;$ Words vs. Faces: $\mathrm{t}(24)=5.942, \mathrm{p}=3.935 \times 10^{-6} ;$ Figure 4A). In contrast, we found no 488 word selectivity in EG's IVWFA (Figure 4B): activation to Words was around baseline and 489 significantly lower than those to Scrambled Words and Objects (Words vs. Scrambled Words:

$490 \mathrm{t}(9)=-3.092, \mathrm{p}=1.289 \times 10^{-2}$; Words vs. Objects: $\left.\mathrm{t}(9)=-2.432, \mathrm{p}=3.785 \times 10^{-2}\right)$ and did not

491 significantly differ from that to Faces (Words vs. Faces: $t(9)=-0.699, p=0.503$ ). Moreover, EG's 492 activation to Words was significantly lower than the control group's $\left(\mathrm{t}(24)=-1.616, \mathrm{p}=5.961 \times 10^{-}\right.$

$492^{2}$, modified t-test; 95\% Bayesian credible interval (CI) $\left.[0.01,0.13]\right)$. This result was stable 494 across thresholds that were used to define the lVWFA fROI (Figure 4C), and we did not observe 495 word selectivity in EG when we searched across the entire lVTC with varying thresholds (Figure 496 3C), or when we used a less stringent contrast (Words vs. Scrambled Words; Supplementary 497 Figure 1). 


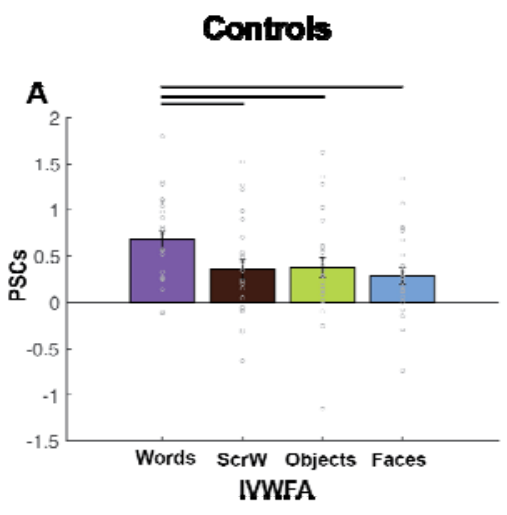

\section{D}

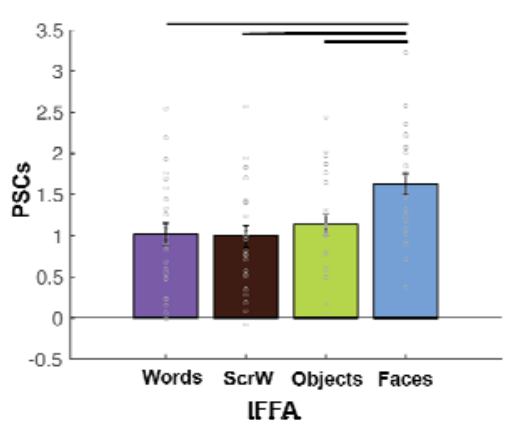

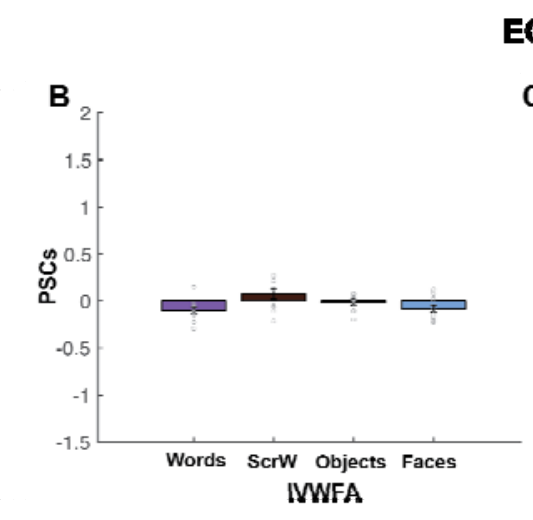

EC

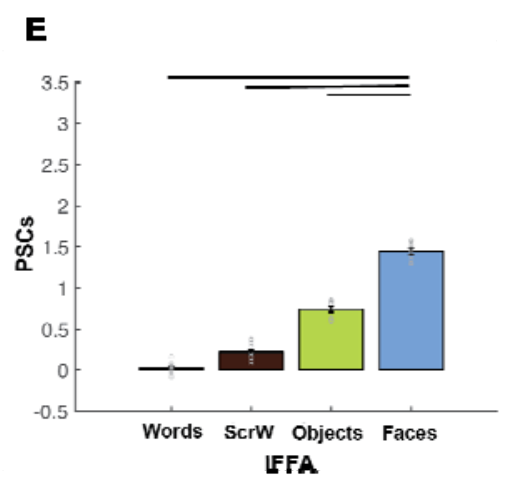

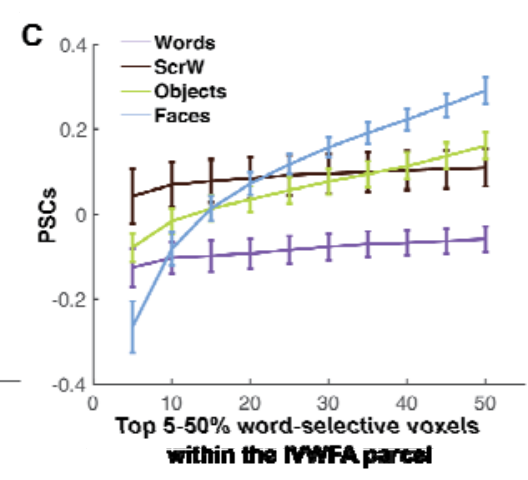

$\mathbf{F}$

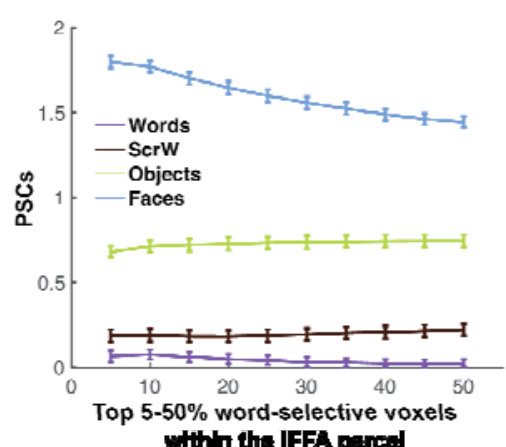

502

503

504

505

506

507

508

509

510

511

512

513

514

515

516

517

518

519

520

521

Figure 4. Responses to four conditions in the IVWFA and IFFA for EG and the controls group. (A), Bar plots show mean PSCs to four conditions estimated in independent data within individually defined IVWFA fROIs (i.e., top 10\% word-selective voxels within lVWFA parcel) for the control group. (B), Mean PSCs to the four conditions estimated in independent data within individually defined IVWFA fROIs for EG. Here and in E, the results are averaged across run combinations.. (C), Parametrically decreasing the threshold for defining the IVWFA fROIs from the top 5\% to 50\% word-selective voxels within the lVWFA parcel. Number of voxels in the defined lVWFA fROIs: 5\%=295 voxels, 50\% $=2402$ voxels. Here and in F, average PSCs across run combinations are shown for each threshold. (D), Mean PSCs across participants to the four conditions estimated in independent data within individually defined IFFA fROIs for the control group. (E), Mean PSCs to four conditions estimated in independent data within individually defined lFFA fROIs for EG. (F), Parametrically decreasing the threshold for defining IFFA fROI from the top 5\% to 50\% face-selective voxels within the lFFA parcel. Number of voxels in the defined lFFA fROIs: $5 \%=18$ voxels, $50 \%=185$ voxels. In the bar plots, dots correspond to individual data points (controls: $\mathrm{n}=25$ subjects; $\mathrm{EG}$ : $\mathrm{n}=10$ run combinations, from ten iterations). Horizontal bars reflect significant paired t-tests $p<0.05$. Error bars in both the bar and line plots denote standard errors of the mean by participants (for the control group) and by run combinations (for EG).

Similar to what we did for the rVTC, to test whether selectivity for other visual categories in the left VTC is typical in EG, we examined face selectivity in EG's lFFA. EG's face selectivity remained intact (Figure 4E) and did not differ from that of the control group (Figure 4D; t(24)=- 
$5230.275, \mathrm{p}=0.393$, modified t-test; 95\% Bayesian CI, [0.27 0.50]). EG's lFFA fROI showed

524 significantly higher responses to Faces than to other conditions (Faces vs. Words: $\mathrm{t}(9)=47.571$,

$525 \mathrm{p}=4.015 \times 10^{-12}$; Faces vs. Scrambled Words: $\mathrm{t}(9)=24.681,1.411 \times 10^{-9}$; Faces vs. Objects:

$526 \mathrm{t}(9)=34.494, \mathrm{p}=7.141 \times 10^{-11}$; Figure 4E), just like what we observed in the control group (Faces

527 vs. Words: $\mathrm{t}(24)=7.076, \mathrm{p}=1.467 \times 10^{-5}$; Faces vs. Scrambled Words: $\mathrm{t}(24)=8.102, \mathrm{p}=2.518 \times 10^{-8}$;

528 Faces vs. Objects: $\mathrm{t}(24)=5.517, \mathrm{p}=1.130 \times 10^{-5}$; Figure 4D). As was the case for the rFFA, the

529 face selectivity in EG's 1FFA was observed across all thresholds used to define the lFFA (Figure

530 4F).

531

532 Altogether, the examination of EG's right and left VTC suggests that without the typical left-

533 hemisphere frontotemporal language network from birth—and presumably without the necessary

534 connections between these areas and parts of the VTC — a canonical VWFA, a word-selective 535 area, does not develop in either hemisphere.

538 Distributed neural representation of visual words: multivariate pattern analysis (MVPA)

540 Our univariate analyses measured the average activation across the voxels most responsive to the

541 visual category in question. Although this is the classic approach to demonstrate category

542 selectivity, univariate analyses may be insensitive to potentially meaningful distributed

543 representation patterns in suprathreshold and/or subthreshold voxels. Previous studies have

544 found that categorical information can be reliably decoded by comparing within-category versus

545 between-category correlations in the VTC (e.g., Haxby et al., 2001). Moreover, a recent study 
546 found mature representational similarity structures via multivariate patterns in children with no

547 univariate selectivity, suggesting that distributed representations may developmentally precede

548 category selectivity (Cohen et al., 2019). To explore whether words may be represented in a

549 distributed fashion in EG, we performed a series of multivariate pattern analyses (MVPA).

551 We first examined the representational similarity matrices (RSMs) in the entire VTC (see

552 Methods) to investigate whether multivariate representational structure for visual categories was

553 preserved in EG. Indeed, we found that EG's rVTC RSM was strongly and significantly

554 correlated with that of the control group $\left(r=0.918, p=9.100 \times 10^{-3}\right.$ (permutation test);

555 Supplementary Figure 3). We tested whether this correlation between EG and the control group

556 was different from the correlations between any given control individual and the rest of the

557 control group (see Methods). Single case comparisons showed that the RSM correlation for EG

558 vs. controls did not significantly differ from the within-controls correlations $(\mathrm{t}(24)=0.797, \mathrm{p}=$

5590.217 , Bayesian 95\% CI [0.09 0.351]). Similar results were found for the lVTC: the RSMs of EG

560 and the control group were strongly and significantly correlated $\left(\mathrm{r}=0.888, \mathrm{p}=7.300 \times 10^{-3}\right)$, and

561 the correlation for EG vs. controls did not significantly differ from the within-controls

562 correlations $(\mathrm{t}(24)=0.835, \mathrm{p}=0.206$, Bayesian 95\% CI [0.09 0.34]).

564 We then asked whether EG's 'VWFA' may contain voxels that show distinct distributed

565 activation patterns to visual words. Specifically, as described in Methods, we searched for voxels

566 that satisfied the following criteria: the searchlight around a given voxel should show 1)

567 distinctive response patterns to Words vs. other categories (e.g., the Words-Words correlation

568 should be higher than the Words-Faces correlation); and 2) stronger within-category correlations 
569 for the preferred category (e.g., the Words-Words correlation should be higher than the Faces-

570 Faces, Objects-Objects, and Scrambled Words-Scrambled Words correlations). Previous studies

571 have shown distributed representations within category-selective regions (e.g., the FFA) of non-

572 preferred categories (e.g., places), and debate is ongoing over whether this information has

573 functional relevance (e.g., Kanwisher, 2010). Our second criterion was included to identify

574 voxels that show more stable multivariate representations for the category of interest (e.g.,

575 Words) compared to other categories. Indeed, we identified a set of voxels within the rVWFA

576 and IVWFA parcels that showed a reliable distributed code for words in both controls and EG

577 (Supplementary Figure 2A, 2C; Supplementary Table 3). In addition, mirroring the univariate

578 analyses, we also identified a set of voxels that showed a reliable distributed code for faces

579 within the rFFA and lFFA parcels in both controls and EG (Supplementary Figure 2B, 2D;

580 Supplementary Table 3).

582 Critically, to test whether these distributed responses to visual words differ between EG and the

583 controls, we subtracted the average within-category correlations for all non-word categories from

584 the within-category correlations for Words. This difference tells us how much stronger the

585 within-category correlation is for the preferred compared to the non-preferred categories. EG

586 showed comparable within-category correlation differences to the controls in both the rVWFA

$587(\mathrm{t}(24)=1.081, \mathrm{p}=0.145$, modified t-test, 95\% Bayesian CI $[0.05,0.26]$ and IVWFA $(\mathrm{t}(24)=0.184$,

$588 \mathrm{p}=0.428$, modified t-test, 95\% Bayesian CI [0.30, 0.50]); Figure 5A). Similar within-category

589 correlation differences were also observed between EG and the controls in the rFFA $(\mathrm{t}(24)=-$

$590 \quad 0.802, \mathrm{p}=0.215 ; 95 \%$ Bayesian CI $[0.09,0.34])$ and $\mathrm{lFFA}(\mathrm{t}(24)=0.488, \mathrm{p}=0.315 ; 95 \%$

591 Bayesian CI [0.18, 0.46]); Figure 5B). 

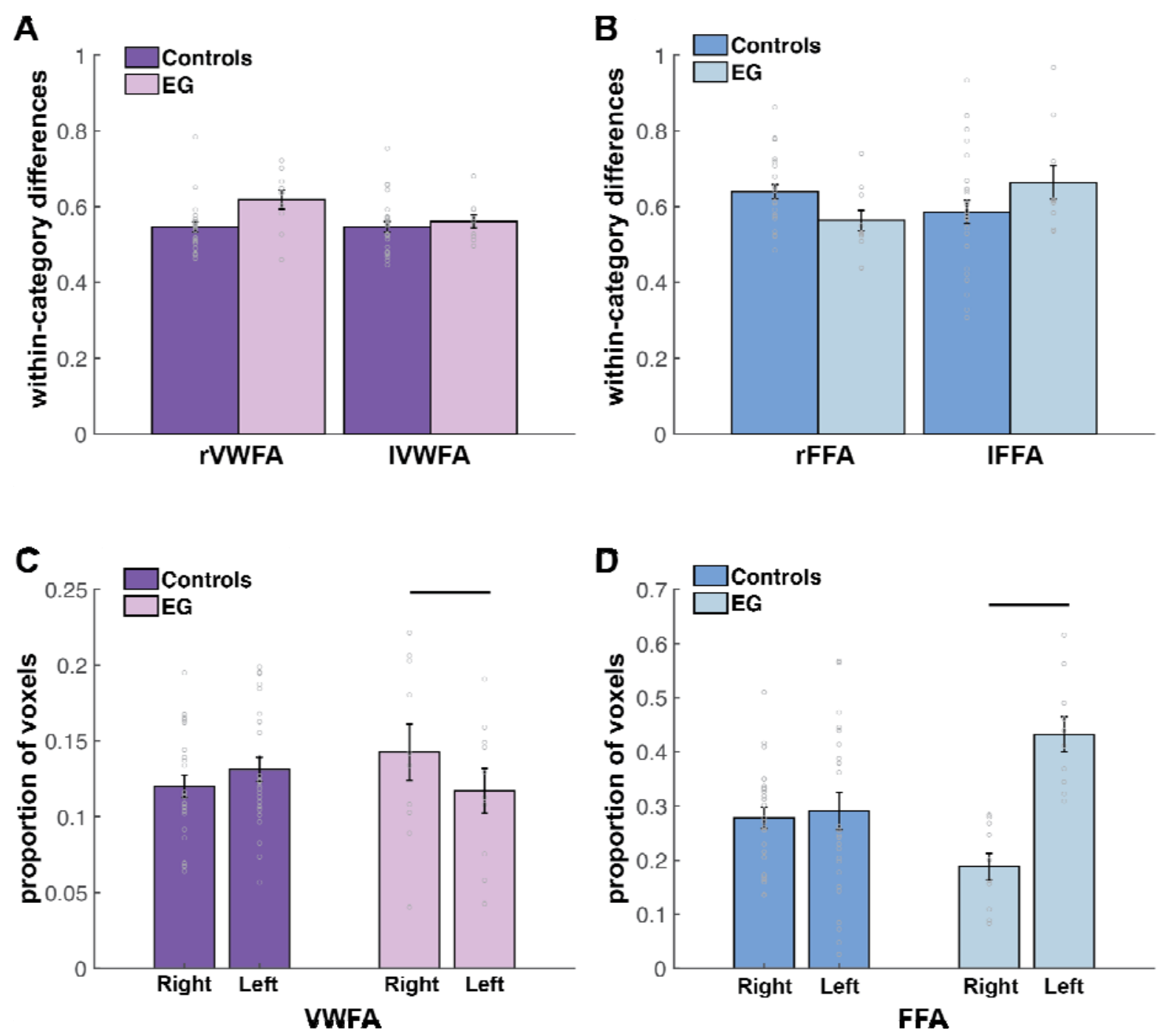

Figure 5. Results of the multivariate pattern analysis (MVPA). (A) Within-category correlation differences between preferred (i.e., Words-Words) and non-preferred (i.e., average within-category correlation of non-word conditions) conditions for EG and the controls in the rVWFA and IVWFA parcels. Here and in B, correlation values were Fisher's z-transformed. (B) Within-category correlation differences between preferred (i.e., Faces-Faces) and non-preferred (i.e., average within-category correlation of non-face conditions) conditions for EG and the controls in the rFFA and lFFA parcels. (C) Proportion of voxels that show multivariate selectivity for Words in the rVWFA and IVWFA parcels for EG and the controls. (D) Proportion of voxels that show multivariate selectivity for Faces in the rFFA and IFFA parcel for EG and the controls. In the bar plots, dots correspond to individual data points (controls: $\mathrm{n}=25$ subjects; EG: $\mathrm{n}=10$ run combinations from ten iterations). Horizontal bars reflect significant LH-RH differences, $\mathrm{p}<0.05$.Error bars denote standard errors of the mean by participants (for the control group) and by run combinations (for EG). 
608 Finally, we explored potential hemispheric asymmetries with respect to multivariate selectivity

609 for words and faces. Left-hemisphere dominance for words and right-hemisphere dominance for

610 faces are well established with univariate measures. Do multivariate patterns also show these

611 asymmetries? We calculated the number of voxels that show multivariate selectivity for words

612 and faces within the left and right VWFA and FFA parcels, respectively (controlling for the size

613 of the search space; see Methods). For Words, mirroring the univariate results in the past

614 literature, a numerically larger proportion of voxels showing multivariate word selectivity was

615 found in the left compared to the right VWFA in the controls (although the difference did not

616 reach significance: $\mathrm{t}(24)=1.181, \mathrm{p}=0.249)$. Interestingly, the opposite pattern was observed in $\mathrm{EG}$,

617 with a larger proportion of voxels showing multivariate word selectivity found in the right

618 VWFA $\left(\mathrm{t}(9)=-2.469, \mathrm{p}=3.564 \times 10^{-2}\right)($ Figure 5C). For Faces, a numerically larger proportion of

619 voxels showing multivariate face selectivity was found in the right compared to the left FFA in

620 the controls (although, like for Words, the difference did not reach significance: $\mathrm{t}(24)=0.367$,

$621 \mathrm{p}=0.717$ ), and EG displayed the opposite pattern: a significantly larger proportion of voxels

622 showing multivariate face selectivity in the left FFA $\left(t(9)=8.983, p=8.672 \times 10^{-6}\right)($ Figure 5D).

\section{Discussion}

627 Case studies provide valuable insights for understanding the functional organization of the

628 human brain and the patterns of reorganization that follow neurological damage (Vaidya et al.,

629 2019). In the current study, we had a unique opportunity to collect fMRI data from an individual

630 (EG) born without her left superior temporal lobe in order to ask whether category-selective 
631 responses to visual words within the ventral temporal cortex would be affected. Specifically, we

632 asked: in the absence of a typical left-hemisphere language network, does word selectivity

633 emerge, and if so, where? Does the VWFA emerge in the right VTC given that EG's language

634 network is reorganized to the right hemisphere (Tuckute et al., 2021)? Or do we instead, or

635 additionally, observe word selectivity in the canonical VWFA within the spared IVTC?

636 Surprisingly, we found no univariate word selectivity within the rVWFA, the right homotope of

637 the canonical IVWFA, and even in an expanded search space of the entire rVTC. We also did not

638 observe word-selective responses in the IVWFA or in any other spared parts of the lVTC.

639 Moreover, the response magnitude to Words (relative to baseline) was significantly lower in

640 EG's 1VWFA than that of controls. Importantly, this lack of category selectivity was specific to

641 Words: selective responses to Faces remained intact in EG's rFFA and lFFA.

643 Absent univariate word selectivity, we then explored multivariate representations of visual words

644 in EG. We found that EG's VTC showed an overall similar representational structure to that of

645 the control group, and that, like the control group, EG had a set of voxels whose local

646 neighborhoods robustly differentiated between Words and other visual categories in their

647 patterns of response. Critically, these voxels also showed a higher within-category correlation

648 (across runs) for Words compared to the within-category correlations for other categories, and

649 the degree of this 'multivariate selectivity' was similar between EG and the controls.

650 Interestingly, however, in contrast to the typically observed left-hemispheric dominance for

651 words and right-hemispheric dominance for faces, EG had a larger proportion of voxels that

652 showed multivariate selectivity for words in her right than her left VWFA, and a larger 
653 proportion of voxels that showed multivariate selectivity for faces in her left FFA than her right

\section{FFA.}

656 Altogether, the current study suggests that in the absence of a typical left-lateralized language

657 network - at least in cases of early left-hemispheric damage, when language processing is

658 'forced' to the right hemisphere-neural selectivity for visual words is, or at least can be, highly

659 atypical. In our participant with extensive left-hemispheric temporal damage and a right-

660 lateralized language network, we observed no word selectivity in the univariate analyses in either

661 the right or the spared left VTC. The absence of such selectivity, combined with EG's intact

662 reading ability, suggests that successful orthographic processing may depend on a more

663 distributed and more right-lateralized neural representation than that observed in typical literate

664 adults.

666 Canonical univariate word selectivity may not emerge when a left-hemisphere language

\section{7 network is missing from birth}

668 The interesting case of EG allowed us to investigate how visual word processing within the VTC

669 can be affected by a congenital or early left-hemisphere lesion outside of visual cortex. Our

670 results provide the first evidence of atypical visual word selectivity in the VTC when the left-

671 lateralized language cortex is missing, and when the language network consequently develops in

672 the right hemisphere (Tuckute et al., 2021) during the early stages of language learning and prior

673 to learning to read. Our study also suggests that even with some remaining anatomical

674 connections between the spared IVTC and the frontal and temporal areas (presumably via local 
675 U-fibers or remaining long-range fibers), the lVWFA will not emerge at the stereotypical

676 location when the left hemisphere does not support language processing.

678 How does a left language network contribute to the development of the VWFA? One possibility

679 is that in a typical brain, the site of the putative VWFA is predisposed to written scripts via

680 intrinsic co-activation with speech and/or language/semantic areas in the left temporal (and

681 maybe frontal) cortex. The processing of speech sounds is tightly linked to reading, and impaired

682 coding of speech sounds (e.g., phonological awareness) is often considered a precursor of

683 dyslexia (e.g., Pennington \& Bishop, 2008; Shaywitz et al., 2002). In the absence of regions that

684 typically support speech processing (within the left superior and middle temporal gyri (STG and

685 MTG); Raschle et al., 2012), EG's left VTC lacked early interactions with these regions. Further,

686 a typical IVWFA communicates visual orthographic information to higher-level left temporal

687 (and maybe frontal) regions that integrate visual and auditory information, like the amodal

688 language regions that process lexical-semantic and combinatorial linguistic information (e.g.,

689 Fedorenko et al., 2020) and the regions that support abstract conceptual processing (e.g., Binder

690 et al., 2009; Patterson et al., 2007)—both sets of areas that were also missing in EG's left

691 hemisphere. Consistent with the idea that connections between the left VTC and the ipsilateral

692 high-level language areas may be critical for the emergence of the lVWFA, a recent study found

693 that in newborns, the putative site of the lVWFA already shows preferential functional

694 connectivity to the areas that will later respond to high-level linguistic information, compared to

695 adjacent regions (Li et al., 2020); this pre-existing functional coupling may further strengthen

696 during language and reading acquisition. Because EG was missing both i) speech-responsive

697 areas, and ii) higher-level language/conceptual areas in her left hemisphere, we cannot evaluate 
698 the relative contributions of these two sets of areas and their connections with the VTC to the

699 emergence of a canonical VWFA. We speculate that both may be important.

Right-hemispheric neural correlates that support visual word processing

702 Because EG's language network resides in the right hemisphere (Tuckute et al., 2021), we

703 expected to find a VWFA in the right VTC. Surprisingly, no univariate word selectivity was

704 observed in EG's VTC, and this result could not be explained by either a lack of functional

705 specialization anywhere in the rVTC (intact face selectivity was observed), or our particular

706 procedure for defining the VWFA (the results held across a range of thresholds, when using a

707 larger search space, and across different contrasts). Interestingly, neurotypical individuals with

708 right-dominant language activation sometimes also lack a VWFA in the right VTC (e.g., Cai et

709 al., 2008; Van der Haegen et al., 2012); in these individuals, IVTC appears to be engaged during

710 word recognition, presumably due to stronger frontotemporal anatomical connections in the LH

711 than in the RH, and any language activation on the left (even if it's non-dominant) would engage

712 the IVTC for reading (Powell et al., 2006). In the case of EG, her right-hemisphere language

713 network may have lacked early interactions with the rVTC, and her left-hemisphere language

714 network was altogether lacking, resulting in the atypical word selectivity (in both right and left)

715 that we observed here.

717 Some have also argued that the development of word-selective cortex directly competes with

718 face-selective cortex for neural resources, thus contributing to right-hemispheric dominance for

719 face processing and left-hemispheric dominance for word processing in neurotypical individuals

720 (e.g., Behrmann \& Plaut, 2015; Dehaene \& Cohen, 2007). Interestingly, EG showed typical face 
721 selectivity in the right FFA, not different from controls. It is therefore possible that a focal word-

722 selective area failed to emerge in EG's rVTC because the relevant cortical tissue had already

723 been 'assigned' to face processing, perhaps due to some specific visual features of faces that are

724 better represented in the right hemisphere/left visual field (e.g., holistic face processing; Li et al.,

725 2017; Rossion et al., 2000). Interestingly, although the univariate response magnitude to faces

726 was higher in EG's right than left FFA (similar to controls), more multivariate-selective face

727 voxels were present in EG's IFFA, compared to her rFFA, in sharp contrast to controls. The

728 functional significance of the latter is at present unclear, but can be explored in future work. On

729 the other hand, EG had more multivariate-selective word voxels in her rVWFA than her IVWFA,

730 presumably related to the fact that her language network resides in the right hemisphere. These

731 data suggest that the hemispheric dominance of the language network drives the laterality of

732 visual processing in the VTC (be it implemented focally, or in a distributed fashion), at least for

733 words, but perhaps also for faces (Behrmann \& Plaut, 2020; Dehaene \& Cohen, 2007).

735 Multivariate responses to words

736 Despite the absence of typical univariate word selectivity, EG's VTC was similar in its overall

737 representation similarity structure - across different visual stimulus classes—-to that of the

738 control group. Consistent with our results, Liu et al. (2019) also found typical representational

739 structure in category-selective regions after resections within or outside VTC; in addition, a

740 recent study found mature representational similarity structures in children (5-7 year-olds) with

741 no univariate selectivity, suggesting that distributed representations precede category selectivity

742 (M. A. Cohen et al., 2019). Our results provide another case where a typical multivariate

743 representational structure is observed in the absence of univariate selectivity for words. 
744 Moreover, our results showed that EG (like typical adults) displayed distinctive activation

745 patterns within the general vicinity of both right and left VWFA that could distinguish visual

746 words from other conditions, with higher within- than between-category correlations (Haxby et

747 al., 2001) and more robust (consistent over time) representations of Words than other conditions.

748 Supporting the idea that multivariate selectivity for words may be functionally useful, Stevens et

749 al. (2017) found the VWFA (defined in individual participants using a standard univariate

750 contrast) discriminated words from pseudowords, and did so more strongly than other control

751 regions (e.g., the FFA).

752

753 Numerous questions remain about the multivariate word representations observed in EG. We

754 will highlight two here. First, what is the relationship between univariate and multivariate

755 representations? Univariate selectivity may partially depend on a connectivity scaffold or other

756 genetically defined instructions to determine the location of functional specialized areas (Deen et

757 al., 2017; Kamps et al., 2020; Li et al., 2020). In contrast, distributed representations like those

758 found in EG may rely less on a connectivity scaffold. Do distributed representations reflect a

759 fundamentally different neural code from that associated with focal representations? Some have

760 argued that the answer is no, and that multivariate analyses may simply be more sensitive than

761 univariate approaches given that they consider the heterogeneity of response across voxels within

762 a region as well as potential subthreshold voxels (Davis et al., 2014). On the other hand,

763 multivariate representations may be truly distinct and reflect information derived from bottom-up

764 visual statistics to a greater extent; and thus perhaps in typical development, representational

765 similarity structures precede univariate selectivity (M. A. Cohen et al., 2019) and emergence of

766 univariate selectivity requires experience-dependent interactions with higher-level areas (e.g., the 
767 language cortex in case of the VWFA). Speculating further, perhaps during EG's early stages of

768 language development (while her language system was maturing in the right hemisphere), the

769 right visual cortex lacked the critical early interaction with the language cortex, due to the lack of

770 strong innate connectivity between the two in the right hemisphere (cf. the left hemisphere; e.g.,

771 Li et al., 2020; Powell et al., 2016), resulting in a more distributed representation for visual

772 words.

773

774 And second, how are multivariate representations read out by downstream brain regions for

775 further processing (Williams et al., 2007); specifically, whereas the behavioral relevance of

776 univariate activity has been extensively investigated in both adults and pediatric populations (e.g.,

777 Furl et al., 2011; Grill-Spector et al., 2004; Huang et al., 2014; Song et al., 2015), the behavioral

778 relevance of multivariate representations is still under debate. One study found that performance

779 in a visual search task among different pairs of object categories could be predicted from

780 underlying neural similarity structures (M. A. Cohen et al., 2016). At this point, it is unclear how

781 multivariate representations for words contribute to EG's reading ability, and whether the right-

782 hemisphere representations are more important—questions that will be a focus of future studies.

\section{$784 \quad$ Limitations and future directions}

785 Overall, our study provides unique evidence that in the absence of a typical left-hemisphere

786 language network - at least in cases of early left-hemisphere damage - the canonical word-

787 selective IVWFA may not develop. Some limitations are worth noting. First, our analyses were

788 restricted to the ventral temporal cortex, which was the focus of our investigation and where we

789 had coverage. Although we show that normal reading ability is possible in the absence of focal 
790 selectivity for word processing in the VTC, there may be other pathways or neural structures

791 outside of the VTC that are important for EG's reading ability? For example, Seghier et al. (2012)

792 reported a patient who acquired dyslexia following extensive left ventral occipitotemporal cortex

793 (LvOT) resection, but then regained reading ability; they provided evidence to suggest that the

794 patient's reading ability was now supported via a direct connection between the occipital visual

795 cortex and the left superior temporal sulcus (STS) without the involvement of the ventral visual

796 stream. It is therefore possible that the STS in the language-dominant hemisphere (i.e., the right

797 hemisphere) may support visual word processing through its connections with the occipital

798 cortex. Future work will explore word processing in EG using whole-brain coverage. Second,

799 EG's missing left superior temporal cortex led to right-lateralized speech and language

800 processing. As discussed earlier, it remains unclear whether speech and higher-level language

801 areas contribute equally to the emergence of the lVWFA, and whether temporal language regions

802 may be more important than the frontal ones. Finally, here we reported a single case that sheds

803 new light on the role of speech and language areas in developing a VWFA. Generalization to

804 other, similar cases, and data from other methods like noninvasive brain stimulation will help us

805 better understand the causal role of language cortex in developing visual word selectivity. 
808

809

810

811

812

813

814

815

816

818

819

820

821

822

823

824

825

826

827

828

829

830

\section{References}

Assem, M., Glasser, M. F., Van Essen, D. C., \& Duncan, J. (2020). A Domain-General Cognitive Core Defined in Multimodally Parcellated Human Cortex. Cerebral Cortex, 30(8), 4361-4380. https://doi.org/10.1093/cercor/bhaa023

Baker, C., Liu, J., Wald, L., Kwong, K. K., Benner, T., \& Kanwisher, N. (2007). Visual word processing and experiential origins of functional selectivity in human extrastriate cortex. Proc Natl Acad Sci USA. https://www.pnas.org/content/104/21/9087

Barttfeld, P., Abboud, S., Lagercrantz, H., Adén, U., Padilla, N., Edwards, A. D., Cohen, L., Sigman, M., Dehaene, S., \& Dehaene-Lambertz, G. (2018). A lateral-to-mesial organization of human ventral visual cortex at birth. Brain Structure and Function, 223(7), 3107-3119. https://doi.org/10.1007/s00429-018-1676-3

Behrmann, M., \& Plaut, D. C. (2013). Distributed circuits, not circumscribed centers, mediate visual recognition. Trends in Cognitive Sciences, 17(5), 210-219. https://doi.org/10.1016/j.tics.2013.03.007

Behrmann, M., \& Plaut, D. C. (2015). A vision of graded hemispheric specialization: Graded hemispheric specialization. Annals of the New York Academy of Sciences, 1359(1), 30-46. https://doi.org/10.1111/nyas.12833

Behrmann, M., \& Plaut, D. C. (2020). Hemispheric Organization for Visual Object Recognition: A Theoretical Account and Empirical Evidence. Perception, 49(4), 373-404. https://doi.org/10.1177/0301006619899049

Binder, J. R., Desai, R. H., Graves, W. W., \& Conant, L. L. (2009). Where Is the Semantic System? A Critical Review and Meta-Analysis of 120 Functional Neuroimaging Studies. Cerebral Cortex, 19(12), 2767-2796. https://doi.org/10.1093/cercor/bhp055 
831 Bishop, D. (2003). Test for Reception of Grammar version 2 (TROG-2).

832

833 Blank, I., Kanwisher, N., \& Fedorenko, E. (2014). A functional dissociation between language and multiple-demand systems revealed in patterns of BOLD signal fluctuations. Journal

Bouhali, F., Thiebaut de Schotten, M., Pinel, P., Poupon, C., Mangin, J.-F., Dehaene, S., \& Cohen, L. (2014). Anatomical Connections of the Visual Word Form Area. Journal of Neuroscience, 34(46), 15402-15414. https://doi.org/10.1523/JNEUROSCI.4918-13.2014

843 Cai, Q., Paulignan, Y., Brysbaert, M., Ibarrola, D., \& Nazir, T. A. (2010). The Left Ventral

847 Cohen, L., Martinaud, O., Lemer, C., Lehéricy, S., Samson, Y., Obadia, M., Slachevsky, A., \& Visual Word Processing System. Journal of Cognitive Neuroscience, 20(4), 672-681. https://doi.org/10.1162/jocn.2008.20043 Occipito-Temporal Response to Words Depends on Language Lateralization but Not on Visual Familiarity. Cerebral Cortex, 20(5), 1153-1163. Dehaene, S. (2003). Visual Word Recognition in the Left and Right Hemispheres: Anatomical and Functional Correlates of Peripheral Alexias. Cerebral Cortex, 13(12), 1313-1333. https://doi.org/10.1093/cercor/bhg079 categories is predicted by the representational architecture of high-level visual cortex. Journal of Neurophysiology, 117(1), 388-402. https://doi.org/10.1152/jn.00569.2016 
854 Cohen, M. A., Dilks, D. D., Koldewyn, K., Weigelt, S., Feather, J., Kell, A. JE., Keil, B., Fischl,

855

856

857

858

859

860

861

862

863

864

865

866

867

868

869

870

871

872

873

874

875

B., Zöllei, L., Wald, L., Saxe, R., \& Kanwisher, N. (2019). Representational similarity

precedes category selectivity in the developing ventral visual pathway. NeuroImage, 197, 565-574. https://doi.org/10.1016/j.neuroimage.2019.05.010

Crawford, J. R., \& Garthwaite, P. H. (2007). Comparison of a single case to a control or normative sample in neuropsychology: Development of a Bayesian approach. Cognitive Neuropsychology, 24(4), 343-372. https://doi.org/10.1080/02643290701290146

Crawford, J. R., Garthwaite, P. H., \& Howell, D. C. (2009). On comparing a single case with a control sample: An alternative perspective. Neuropsychologia, 47(13), 2690-2695. https://doi.org/10.1016/j.neuropsychologia.2009.04.011

Crawford, J. R., \& Howell, D. C. (1998). Comparing an Individual's Test Score Against Norms Derived from Small Samples. The Clinical Neuropsychologist, 12(4), 482-486. https://doi.org/10.1076/clin.12.4.482.7241

Davis, T., LaRocque, K. F., Mumford, J. A., Norman, K. A., Wagner, A. D., \& Poldrack, R. A. (2014). What do differences between multi-voxel and univariate analysis mean? How subject-, voxel-, and trial-level variance impact fMRI analysis. NeuroImage, 97, 271-283. https://doi.org/10.1016/j.neuroimage.2014.04.037

Deen, B., Richardson, H., Dilks, D. D., Takahashi, A., Keil, B., Wald, L. L., Kanwisher, N., \& Saxe, R. (2017). Organization of high-level visual cortex in human infants. Nature Communications, 8(1), 13995. https://doi.org/10.1038/ncomms13995

Dehaene, S., \& Cohen, L. (2007). Cultural Recycling of Cortical Maps. Neuron, 56(2), 384-398. https://doi.org/10.1016/j.neuron.2007.10.004 
876 Dehaene, S., \& Cohen, L. (2011). The unique role of the visual word form area in reading. Trends in Cognitive Sciences, 15(6), 254-262. https://doi.org/10.1016/j.tics.2011.04.003

878 Dehaene, S., Cohen, L., Morais, J., \& Kolinsky, R. (2015). Illiterate to literate: Behavioural and cerebral changes induced by reading acquisition. Nature Reviews Neuroscience, 16(4),

881 Dehaene-Lambertz, G., Monzalvo, K., \& Dehaene, S. (2018). The emergence of the visual word form: Longitudinal evolution of category-specific ventral visual areas during reading acquisition. PLOS Biology, 16(3), e2004103. https://doi.org/10.1371/journal.pbio.2004103 automated labeling system for subdividing the human cerebral cortex on MRI scans into gyral based regions of interest. NeuroImage, 31(3), 968-980.

893 Duncan, J., Assem, M., \& Shashidhara, S. (2020). Integrated Intelligence from Distributed Brain Activity. Trends in Cognitive Sciences, 24(10), 838-852. https://doi.org/10.1016/j.neuroimage.2006.01.021

Downing, P. E., Jiang, Y., Shuman, M., \& Kanwisher, N. (2001). A cortical area selective for visual processing of the human body. Science (New York, N.Y.), 293(5539), 2470-2473. https://doi.org/10.1126/science.1063414

896 Dunn, L. M., \& Dunn, D. M. (2007). The Peabody Picture Vocabulary Test, Fourth Edition. 897 NCS

898 Pearson, Inc. 
Epstein, R., \& Kanwisher, N. (1998). A cortical representation of the local visual environment.

900 Nature, 392(6676), 598-601. https://doi.org/10.1038/33402

901 Fedorenko, E., Behr, M. K., \& Kanwisher, N. (2011). Functional specificity for high-level linguistic processing in the human brain. Proceedings of the National Academy of

904 François, C., Garcia-Alix, A., Bosch, L., \& Rodriguez-Fornells, A. (2021). Signatures of brain plasticity supporting language recovery after perinatal arterial ischemic stroke. Brain and Language, 212, 104880. https://doi.org/10.1016/j.bandl.2020.104880

907 Furl, N., Garrido, L., Dolan, R. J., Driver, J., \& Duchaine, B. (2011). Fusiform Gyrus Face Selectivity Relates to Individual Differences in Facial Recognition Ability. Journal of Cognitive Neuroscience, 23(7), 1723-1740. https://doi.org/10.1162/jocn.2010.21545

910 Gerrits, R., Van der Haegen, L., Brysbaert, M., \& Vingerhoets, G. (2019). Laterality for recognizing written words and faces in the fusiform gyrus covaries with language

913 Glezer, L. S., Jiang, X., \& Riesenhuber, M. (2009). Evidence for Highly Selective Neuronal

914 Tuning to Whole Words in the "Visual Word Form Area." Neuron, 62(2), 199-204. 915 https://doi.org/10.1016/j.neuron.2009.03.017

916 Greenblatt, S. H. (1976). Subangular alexia without agraphia or hemianopsia. Brain and 917 Language, 3(2), 229-245. https://doi.org/10.1016/0093-934X(76)90019-5

918 Grill-Spector, K., Knouf, N., \& Kanwisher, N. (2004). The fusiform face area subserves face 919 perception, not generic within-category identification. Nature Neuroscience, 7(5), 555920 562. https://doi.org/10.1038/nn1224 
921 Hamamé, C. M., Szwed, M., Sharman, M., Vidal, J. R., Perrone-Bertolotti, M., Kahane, P.,

922 Bertrand, O., \& Lachaux, J.-P. (2013). Dejerine’s reading area revisited with intracranial

923 EEG: Selective responses to letter strings. Neurology, 80(6), 602-603.

924 https://doi.org/10.1212/WNL.0b013e31828154d9

925 Hannagan, T., Amedi, A., Cohen, L., Dehaene-Lambertz, G., \& Dehaene, S. (2015). Origins of

926 the specialization for letters and numbers in ventral occipitotemporal cortex. Trends in

$927 \quad$ Cognitive Sciences, 19(7), 374-382. https://doi.org/10.1016/j.tics.2015.05.006

928 Hasson, U., Levy, I., Behrmann, M., Hendler, T., \& Malach, R. (2002). Eccentricity Bias as an

929 Organizing Principle for Human High-Order Object Areas. Neuron, 34(3), 479-490.

930 https://doi.org/10.1016/S0896-6273(02)00662-1

931 Haxby, J. V., Gobbini, M. I., Furey, M. L., Ishai, A., Schouten, J. L., \& Pietrini, P. (2001).

932 Distributed and Overlapping Representations of Faces and Objects in Ventral Temporal

933 Cortex. Science, 293(5539), 2425-2430. https://doi.org/10.1126/science.1063736

934 Hsiao, J. H., \& Lam, S. M. (2013). The Modulation of Visual and Task Characteristics of a

935 Writing System on Hemispheric Lateralization in Visual Word Recognition-A

936 Computational Exploration. Cognitive Science, 37(5), 861-890.

937 https://doi.org/10.1111/cogs.12033

938 Huang, L., Song, Y., Li, J., Zhen, Z., Yang, Z., \& Liu, J. (2014). Individual differences in

939 cortical face selectivity predict behavioral performance in face recognition. Frontiers in

$940 \quad$ Human Neuroscience, 8. https://doi.org/10.3389/fnhum.2014.00483

941 Julian, J. B., Fedorenko, E., Webster, J., \& Kanwisher, N. (2012). An algorithmic method for

942 functionally defining regions of interest in the ventral visual pathway. NeuroImage, 60(4),

943 2357-2364. https://doi.org/10.1016/j.neuroimage.2012.02.055 
944 Kamps, F. S., Hendrix, C. L., Brennan, P. A., \& Dilks, D. D. (2020). Connectivity at the origins

945 of domain specificity in the cortical face and place networks. Proceedings of the National

946 Academy of Sciences, 117(11), 6163-6169. https://doi.org/10.1073/pnas.1911359117

947 Kanwisher, N. (2010). Functional specificity in the human brain: A window into the functional

948 architecture of the mind. Proceedings of the National Academy of Sciences, 107(25),

949 11163-11170. https://doi.org/10.1073/pnas.1005062107

950 Kanwisher, N., McDermott, J., \& Chun, M. M. (1997). The Fusiform Face Area: A Module in

951 Human Extrastriate Cortex Specialized for Face Perception. The Journal of Neuroscience,

952 17(11), 4302-4311. https://doi.org/10.1523/JNEUROSCI.17-11-04302.1997

953 Kaufman, A. S., \& Kaufman, N. L. (2004). Kaufman Brief Intelligence Test (2nd ed.). American

$954 \quad$ Guidance Service.

955 Kay, J., Coltheart, M., \& Lesser, R. (1992). Psycholinguistic assessments of language processing

956 in aphasia (PALPA) (1st Edition). Psychology Press.

957 Konkle, T., \& Caramazza, A. (2013). Tripartite Organization of the Ventral Stream by Animacy

958 and Object Size. Journal of Neuroscience, 33(25), 10235-10242.

959 Lerma-Usabiaga, G., Carreiras, M., \& Paz-Alonso, P. M. (2018). Converging evidence for

960 functional and structural segregation within the left ventral occipitotemporal cortex in

961 reading. Proceedings of the National Academy of Sciences, 115(42), E9981-E9990.

962 https://doi.org/10.1073/pnas.1803003115

963 Li, J., Huang, L., Song, Y., \& Liu, J. (2017). Dissociated neural basis of two behavioral

964 hallmarks of holistic face processing: The whole-part effect and composite-face effect.

965 Neuropsychologia, 102, 52-60. https://doi.org/10.1016/j.neuropsychologia.2017.05.026 
966

967

968

969

970

971

972

973

974

975

976

977

978

979

980

981

982

983

984

985

986

987

Li, J., Osher, D. E., Hansen, H. A., \& Saygin, Z. M. (2020). Innate connectivity patterns drive the development of the visual word form area. Scientific Reports, 10(1), 18039. https://doi.org/10.1038/s41598-020-75015-7

Liu, T. T., Freud, E., Patterson, C., \& Behrmann, M. (2019). Perceptual Function and CategorySelective Neural Organization in Children with Resections of Visual Cortex. Journal of Neuroscience, 39(32), 6299-6314. https://doi.org/10.1523/JNEUROSCI.3160-18.2019

Mahon, B. Z., \& Caramazza, A. (2011). What drives the organization of object knowledge in the brain? Trends in Cognitive Sciences, 15(3), 97-103. https://doi.org/10.1016/j.tics.2011.01.004

Malach, R., Levy, I., \& Hasson, U. (2002). The topography of high-order human object areas. Trends in Cognitive Sciences, 6(4), 176-184. https://doi.org/10.1016/S13646613(02)01870-3

Martin, A. (2006). Shades of Déjerine-Forging a Causal Link between the Visual Word Form Area and Reading. Neuron, 50(2), 173-175. https://doi.org/10.1016/j.neuron.2006.04.004

Mbwana, J., Berl, M. M., Ritzl, E. K., Rosenberger, L., Mayo, J., Weinstein, S., Conry, J. A., Pearl, P. L., Shamim, S., Moore, E. N., Sato, S., Vezina, L. G., Theodore, W. H., \& Gaillard, W. D. (2009). Limitations to plasticity of language network reorganization in localization related epilepsy. Brain, 132(2), 347-356. https://doi.org/10.1093/brain/awn329

McCandliss, B. D., Cohen, L., \& Dehaene, S. (2003). The visual word form area: Expertise for reading in the fusiform gyrus. Trends in Cognitive Sciences, 7(7), 293-299. https://doi.org/10.1016/S1364-6613(03)00134-7 
Morais, J., Cary, L., Alegria, J., \& Bertelson, P. (1979). Does awareness of speech as a sequence of phones arise spontaneously? Cognition, 7(4), 323-331. https://doi.org/10.1016/00100277(79)90020-9

Nasr, S., Echavarria, C. E., \& Tootell, R. B. H. (2014). Thinking Outside the Box: Rectilinear Shapes Selectively Activate Scene-Selective Cortex. Journal of Neuroscience, 34(20), 6721-6735. https://doi.org/10.1523/JNEUROSCI.4802-13.2014

Newport, E. L., Landau, B., Seydell-Greenwald, A., Turkeltaub, P. E., Chambers, C. E., Dromerick, A. W., Carpenter, J., Berl, M. M., \& Gaillard, W. D. (2017). Revisiting Lenneberg's Hypotheses About Early Developmental Plasticity: Language Organization After Left-Hemisphere Perinatal Stroke. Biolinguistics, 11, 407-422.

Oosterhof, N. N., Connolly, A. C., \& Haxby, J. V. (2016). CoSMoMVPA: Multi-Modal Multivariate Pattern Analysis of Neuroimaging Data in Matlab/GNU Octave. Frontiers in Neuroinformatics, 10. https://doi.org/10.3389/fninf.2016.00027

Osher, D. E., Saxe, R., Koldewyn, K., Gabrieli, J. D. E., Kanwisher, N., \& Saygin, Z. M. (2015). Structural Connectivity Fingerprints Predict Cortical Selectivity for Multiple Visual Categories across Cortex. Cerebral Cortex, 26(4), 1668-1683.

Patterson, K., Nestor, P. J., \& Rogers, T. T. (2007). Where do you know what you know? The representation of semantic knowledge in the human brain. Nature Reviews Neuroscience, 8(12), 976-987. https://doi.org/10.1038/nrn2277

Pennington, B. F., \& Bishop, D. V. M. (2008). Relations Among Speech, Language, and Reading Disorders. Annual Review of Psychology, 60(1), 283-306. https://doi.org/10.1146/annurev.psych.60.110707.163548 
1010 Postelnicu, G., Zollei, L., \& Fischl, B. (2009). Combined Volumetric and Surface Registration.

1011

1012

1013

1014

1015

1016

1017

1018

1019

1020

1021

1022

1023

1024

1025

1026

1027

1028

1029

1030

1031

1032

IEEE Transactions on Medical Imaging, 28(4), 508-522.

https://doi.org/10.1109/TMI.2008.2004426

Powell, H. W. R., Parker, G. J. M., Alexander, D. C., Symms, M. R., Boulby, P. A., WheelerKingshott, C. A. M., Barker, G. J., Noppeney, U., Koepp, M. J., \& Duncan, J. S. (2006).

Hemispheric asymmetries in language-related pathways: A combined functional MRI and tractography study. NeuroImage, 32(1), 388-399.

https://doi.org/10.1016/j.neuroimage.2006.03.011

Raschle, N. M., Zuk, J., \& Gaab, N. (2012). Functional characteristics of developmental dyslexia in left-hemispheric posterior brain regions predate reading onset. Proceedings of the National Academy of Sciences, 109(6), 2156-2161. https://doi.org/10.1073/pnas.1107721109

Rauschecker, A. M., Deutsch, G. K., Ben-Shachar, M., Schwartzman, A., Perry, L. M., \& Dougherty, R. F. (2009). Reading impairment in a patient with missing arcuate fasciculus. Neuropsychologia, 47(1), 180-194. https://doi.org/10.1016/j.neuropsychologia.2008.08.011

Rosenke, M., van Hoof, R., van den Hurk, J., Grill-Spector, K., \& Goebel, R. (2021). A Probabilistic Functional Atlas of Human Occipito-Temporal Visual Cortex. Cerebral Cortex, 31(1), 603-619. https://doi.org/10.1093/cercor/bhaa246

Rossion, B., Dricot, L., Devolder, A., Bodart, J.-M., Crommelinck, M., Gelder, B. de, \& Zoontjes, R. (2000). Hemispheric Asymmetries for Whole-Based and Part-Based Face Processing in the Human Fusiform Gyrus. Journal of Cognitive Neuroscience, 12(5), 793-802. https://doi.org/10.1162/089892900562606 
1033 Saygin, Z. M., Osher, D. E., Koldewyn, K., Reynolds, G., Gabrieli, J. D. E., \& Saxe, R. (2012).

1034 Anatomical connectivity patterns predict face selectivity in the fusiform gyrus. Nature

$1035 \quad$ Neuroscience, 15, 321-327.

1036 Saygin, Z. M., Osher, D. E., Norton, E. S., Youssoufian, D. A., Beach, S. D., Feather, J., Gaab, N., Gabrieli, J. D. E., \& Kanwisher, N. (2016). Connectivity precedes function in the development of the visual word form area. Nature Neuroscience, 19(9), 1250-1255. https://doi.org/10.1038/nn.4354

Seghier, M. L., Neufeld, N. H., Zeidman, P., Leff, A. P., Mechelli, A., Nagendran, A., Riddoch, J. M., Humphreys, G. W., \& Price, C. J. (2012). Reading without the left ventral occipitotemporal cortex. Neuropsychologia, 50(14), 3621-3635. https://doi.org/10.1016/j.neuropsychologia.2012.09.030

Seghier, M. L., \& Price, C. J. (2011). Explaining Left Lateralization for Words in the Ventral Occipitotemporal Cortex. Journal of Neuroscience, 31(41), 14745-14753. https://doi.org/10.1523/JNEUROSCI.2238-11.2011

Song, Y., Zhu, Q., Li, J., Wang, X., \& Liu, J. (2015). Typical and Atypical Development of Functional Connectivity in the Face Network. Journal of Neuroscience, 35(43), 1462414635. https://doi.org/10.1523/JNEUROSCI.0969-15.2015

Staudt, M. (2010). Reorganization after pre- and perinatal brain lesions*. Journal of Anatomy, 217(4), 469-474. https://doi.org/10.1111/j.1469-7580.2010.01262.x 
1056 Staudt, M., Grodd, W., Niemann, G., Wildgruber, D., Erb, M., \& Krägeloh-Mann, I. (2001).

1057

1058

1059

1060

1061

1062

1063

1064

1065

1066

1067

1068

1069

1070

1071

1072

1073

1074

1075

1076

1077

1078

Early left periventricular brain lesions induce right hemispheric organization of speech. Neurology, 57(1), 122-125. https://doi.org/10.1212/WNL.57.1.122

Stevens, W. D., Kravitz, D. J., Peng, C. S., Tessler, M. H., \& Martin, A. (2017). Privileged Functional Connectivity between the Visual Word Form Area and the Language System. The Journal of Neuroscience, 37(21), 5288-5297. https://doi.org/10.1523/JNEUROSCI.0138-17.2017

Tadros, K., Dupuis-Roy, N., Fiset, D., Arguin, M., \& Gosselin, F. (2013). Reading laterally: The cerebral hemispheric use of spatial frequencies in visual word recognition. Journal of Vision, 13(1), 4-4. https://doi.org/10.1167/13.1.4

Tuckute, G., Paunov, A., Kean, H., Small, H., Mineroff, Z., Blank, I., \& Fedorenko, E. (2021). Frontal language areas do not emerge in the absence of temporal language areas: A case study of an individual born without a left temporal lobe. BioRxiv, 2021.05.28.446230. https://doi.org/10.1101/2021.05.28.446230

Vaidya, A. R., Pujara, M. S., Petrides, M., Murray, E. A., \& Fellows, L. K. (2019). Lesion Studies in Contemporary Neuroscience. Trends in Cognitive Sciences, 23(8), 653-671. https://doi.org/10.1016/j.tics.2019.05.009

Van der Haegen, L., Cai, Q., \& Brysbaert, M. (2012). Colateralization of Broca's area and the visual word form area in left-handers: FMRI evidence. Brain and Language, 122(3), 171-178. https://doi.org/10.1016/j.bandl.2011.11.004

Wandell, B. A., Rauschecker, A. M., \& Yeatman, J. D. (2012). Learning to See Words. Annual Review of Psychology, 63(1), 31-53. https://doi.org/10.1146/annurev-psych-120710100434 
1079 Williams, M. A., Dang, S., \& Kanwisher, N. G. (2007). Only some spatial patterns of fMRI

1080 response are read out in task performance. Nature Neuroscience, 10(6), 685-686.

1081 https://doi.org/10.1038/nn1900

1082 Wilmer, J. B., Germine, L., Chabris, C. F., Chatterjee, G., Williams, M., Loken, E., Nakayama,

1083 K., \& Duchaine, B. (2010). Human face recognition ability is specific and highly

1084 heritable. Proceedings of the National Academy of Sciences, 107(11), 5238-5241.

1085 https://doi.org/10.1073/pnas.0913053107

1086 Yeatman, J. D., \& Feldman, H. M. (2013). Neural plasticity after pre-linguistic injury to the

1087 arcuate and superior longitudinal fasciculi. Cortex, 49(1), 301-311.

1088 https://doi.org/10.1016/j.cortex.2011.08.006

1089 Zhu, Q., Song, Y., Hu, S., Li, X., Tian, M., Zhen, Z., Dong, Q., Kanwisher, N., \& Liu, J. (2010).

1090 Heritability of the Specific Cognitive Ability of Face Perception. Current Biology, 20(2),

1091 137-142. https://doi.org/10.1016/j.cub.2009.11.067

1092 